British Journal of Nutrition (2022), 128, 1832-1847

doi: $10.1017 /$ S0007114521004724

(C) The Author(s), 2021. Published by Cambridge University Press on behalf of The Nutrition Society. This is an Open Access article, distributed under the terms of the Creative Commons Attribution-NonCommercial-NoDerivatives licence (https://creativecommons.org/licenses/by-nc-nd/4.0/), which permits non-commercial re-use, distribution, and reproduction in any medium, provided the original work is unaltered and is properly cited. The written permission of Cambridge University Press must be obtained for commercial re-use or in order to create a derivative work.

\title{
Food insecurity, low dietary diversity and poor mental health among Syrian refugee mothers living in vulnerable areas of Greater Beirut, Lebanon
}

\author{
Joana Abou-Rizk ${ }^{1 *}$, Theresa Jeremias ${ }^{1}$, Georgiana Cocuz ${ }^{1}$, Lara Nasreddine ${ }^{2}$, Lamis Jomaa ${ }^{2}$, Nahla Hwalla ${ }^{2}$, \\ Jan Frank ${ }^{1}$ and Veronika Scherbaum ${ }^{1}$ \\ ${ }^{1}$ Institute of Nutritional Sciences (140), University of Hohenheim, Stuttgart, Germany \\ ${ }^{2}$ Department of Nutrition and Food Sciences, American University of Beirut, Beirut, Lebanon
}

(Submitted 6 June 2021 - Final revision received 8 November 2021 - Accepted 25 November 2021 - First published online 29 November 2021)

Abstract

Syrian refugees in Lebanon are facing vulnerabilities that are affecting their food insecurity (FI) levels. The objectives of this study were to measure dietary diversity, FI and mental health status of Syrian refugee mothers in Lebanon and to explore its associations with their anaemia and nutritional status. A cross-sectional study was conducted among mothers with children under 5 years $(n$ 433) in Greater Beirut, Lebanon. Dietary diversity was measured using the Minimum Dietary Diversity for Women (MDD-W) of reproductive age and FI using the global Food Insecurity Experience Scale at the individual level. Depression and post-traumatic stress disorder (PTSD) were measured to assess the maternal mental health status. Data on socio-economic characteristics, anthropometric measurements and $\mathrm{Hb}$ concentrations were collected. Overall, $63.3 \%$ of the mothers had a low dietary diversity (LDD) and $34.4 \%$ were moderately to severely food insecure, with $12.5 \%$ being severely food insecure. The prevalence of PTSD, moderate depression and severe depression was 13.2, 11.1 and 9.9\%, respectively. A significant correlation was found between LDD and FI $(P<0 \cdot 001)$. Low income was significantly associated with LDD and FI. Poor mental health was significantly associated with FI. LDD and FI were not associated with anaemia and nutritional status of mothers. Low-income households had significantly higher intakes of grains and refined starchy staples, whereas high-income households consumed more nutritious foods and sweets. Evidence of inadequate diet quality, FI and poor mental health among Syrian refugee mothers in Lebanon is presented. Multifaceted actions are needed to reduce FI and improve dietary diversity.

\section{Key words: Dietary diversity: Food insecurity: Food consumption: Mental health: Syrian refugees: Lebanon}

The eradication of hunger, achieving food security and promoting adequate nutrition, is the ultimate aim of the Sustainable Development Goal 2, yet it remains a major challenge worldwide ${ }^{(1)}$. Since 1996, food security is declared a human right and 'exists when all people, at all times, have physical and economic access to sufficient, safe and nutritious food to meet their dietary needs and food preferences for an active and healthy life ${ }^{\text {(2) }}$. Food insecurity (FI) and malnutrition continue to affect hundreds of million people and may be both a cause and a consequence of conflicts and protracted crises $^{(3-5)}$. While hunger may be associated with severe FI, moderate FI reflects uncertainties about the ability to obtain food and may compromise diet quality and diversity ${ }^{(6,7)}$

Food-coping strategies may be employed to decrease food consumption in the face of emerging shortfalls ${ }^{(8)}$. Households may resort to rationing strategies such as reducing the number of meals, limiting portion sizes, restricting consumption by adults in favour of children or even skipping entire days without eating. Other strategies involve dietary changes such as eating fewer kinds of food groups and relying on less preferred or inexpensive foods ${ }^{(9)}$. As households and individuals may attempt to cope with household FI, women may be particularly consuming a more monotonous diet and be exposed to a higher risk of malnutrition due to the maternal buffering to feed children first, their roles within households and sex inequalities within societies. Intrahousehold differences in food allocation and choices may be masked by the measure of FI at the household level, indicating a need to assess at the individual level ${ }^{(7)}$. In fact, the perception of the concept of 'being out of food' is different between men and women. While men tend to interpret it as not being able to feed the family, women associate it more with a monotonous $\operatorname{diet}^{(10)}$.

Poor dietary diversity has an exacerbating effect on the nutritional status of children and women of reproductive

Abbreviations: EAR, estimated average requirement; \% EI, percentage energy intake; FI, food insecurity; FIES, Food Insecurity Experience Scale; LDD, low dietary diversity; LM, lactating mother; MDD-W, Minimum Dietary Diversity for Women; NPNLM, non-pregnant non-lactating mother; PHCC, primary healthcare centre; PM, pregnant mother; PTSD, post-traumatic stress disorder; WFP, World Food Programme. 
age, particularly pregnant mother (PM) and lactating mother $(\mathrm{LM})^{(6,11)}$. Proper nutrition during the first $1000 \mathrm{~d}$, since conception to the child's second birthday, lays the foundations for optimal growth and healthy life for more than just a generation ${ }^{(12-14)}$. Paradoxically, undernutrition and overweight can both emerge from FI and poverty ${ }^{(7)}$. This paradox exists due to the compromise in the diet quality by consuming foods with higher energy density, often less expensive, rather than foods with higher nutrient density, which contributes to nutrient inadequacies and excessive energy intake ${ }^{(15,10)}$. Furthermore, FI and depression have been previously linked in many contexts ${ }^{(17)}$. Feelings of deprivation, anxiety and poor mental health may emerge due to the struggle to provide food regularly in the context of poverty ${ }^{(7,18)}$. Poor maternal mental health may ultimately have a negative effect on caregiving and the child's development, especially in low socio-economic contexts ${ }^{(19-21)}$.

More than a decade ago, the Arab Spring surged in the Middle East and North Africa region with poverty and FI being the main drivers and outcomes of the protests and wars that followed ${ }^{(22,23)}$.

The Syrian war, a protracted conflict that is currently in its eleventh year, remains one of the major challenges of the twenty-first century, with more than 5.5 million refugees still residing in neighbouring countries, including Lebanon, Turkey, Jordan, Egypt and $\operatorname{Iraq}^{(23,24)}$. Lebanon hosts more than 1.5 million Syrians and remains the country with the highest refugee per capita concentration worldwide ${ }^{(25)}$. Despite large-scale assistance programmes, about half of the Syrian refugee households in Lebanon were living in extreme poverty in 2018 and 2019. They continue to face tremendous challenges that affect their FI status and increase vulnerabilities associated with their legal status, restricted mobility, access to services and limited employment opportunities ${ }^{(26)}$. With few informal settlements in Greater Beirut, the vast majority of Syrian refugees are living in rented accommodation ${ }^{(27)}$ and are suffering from high costs of living, household debts and dependency on food assistance (e-vouchers). Recent findings among Syrian refugee mothers in Greater Beirut showed that high levels of overweight and obesity coexist with anaemia, in addition to poor maternal diet and nutritional inadequacies ${ }^{(28)}$.

Despite numerous surveys conducted annually by the UN High Commission on Refugees (UNHCR) and the World Food Programme (WFP) on the household level of FI and food consumption among Syrian refugees in Lebanon ${ }^{(25)}$, limited data explore FI at the individual level and dietary diversity among female refugees in urban settings of a humanitarian crisis. The present study aims to: (1) assess the prevalence of FI, maternal low dietary diversity (LDD) and poor mental health of Syrian refugee mothers of children under 5 years, (2) explore the associations between dietary diversity, FI, mental health, anaemia and nutritional status of mothers and (3) examine the consumption food groups according to household monthly income among Syrian refugee mothers in Greater Beirut following years into the crisis.

\section{Methods}

\section{Study design and sampling method}

A cross-sectional survey was carried out in July-September 2018 among Syrian refugee mothers with at least one child below
5 years living in vulnerable areas of Greater Beirut, Lebanon. A two-step purposeful sampling was used to select primary healthcare centres (PHCC) in localities in Greater Beirut with the highest vulnerability level ${ }^{(29)}$, which included Baouchriyeh, Bourj Barajneh, Bourj Hammoud, Chiyah, Mazraa and Mousaytbeh. Greater Beirut is considered to be the melting pot of the country; therefore, the catchment area of PHCC in Greater Beirut covers the capital city Beirut and adjacent urban surroundings of Mount Lebanon Governorate ${ }^{(30)}$. Inclusion criteria included women of reproductive age (15-49 years) from Syrian households with at least one child (0-59 months) that is not suffering from any inborn errors of metabolism or physical malformations. Mother-child dyads were identified through three approaches, via: (1) the nurses at PHCC, (2) direct contact by the research assistant in the waiting rooms and (3) flyers with a brief description of the study made available in PHCC locations. A detailed methodology of this survey has been described elsewhere ${ }^{(28)}$.

The present study is part of a larger cross-sectional survey among Syrian refugee mothers and their children under 5 years in Greater Beirut that explored anaemia as one of the key outcomes. Sample size calculations were based on previous estimates of anaemia of $26.1 \%$ among Syrian refugee women in Lebanon ${ }^{(31)}$. A minimum of 296 participants were needed to provide a power of $80 \%$ and a margin of error of $5 \%$ at $95 \%$ CI. Accounting for a study design effect of 1.5 , non-response rate of $15 \%$ and dropout rate of $10 \%$, we aimed for a sample size of 555 mothers. Out of the 665 women approached, 590 mothers with at least one child under the age of 5 years from Syrian households were eligible and 489 mothers (82.9\%) consented to participate in the survey. A total of 433 mothers ( $88.6 \%$ ) completed the interview and were included in the present study.

\section{Ethical approval and consent to participate}

The original study was approved by the Institutional Review Board for Social and Behavioral studies at the American University of Beirut in Lebanon (IRB ID: SBS-2017-0294) and the Freiburg Ethics Commission International in Germany (FEKI code: 017/1434). The Primary Health Care (PHC) Department at the Ministry of Public Health (MoPH) granted with an approval letter access to PHCC in Greater Beirut, which is part of the National PHC Network overseen by the MoPH. Directors of PHCC selected for the survey also granted approval letters prior accessing their premises. Consent forms and questionnaires written in North Levantine Arabic dialect were used. Written informed consent was obtained prior enrolment in the study, and a copy of the consent form was given to each participant. A parental consent and an informed assent were sought from mothers aged below 18 years. In case of illiteracy, a witness or the nurse on duty signed on the behalf of the participant after reading and explaining the consent form. Confidentiality and voluntary participation were assured by the data collectors by assigning random identifiers and allowing access to the data only to the investigators.

\section{Data collection}

A culture-specific multi-component questionnaire was carried out during face-to-face interviews by Collaborative Institutional 
Training Initiative certified and well-trained enumerators. Data quality control was performed on a regular basis to ensure an increased accuracy of data and to limit risks of reporting bias. Data on socio-economic and household characteristics such as parents' education level and employment status, household monthly income, size of the household, registration with the UNHCR as refugees, receiving food assistance (e-vouchers) from the WFP and healthcare services utilisation were collected. Mental health status of mothers was assessed using the Patient Health Questionnaire-9 to measure depression ${ }^{(32)}$, validated among Lebanese adults $^{(33)}$ and the Arabic version of the Mini International Neuropsychiatric Interview (MINI) to measure post-traumatic stress disorder (PTSD) ${ }^{(34)}$. Crowding index was defined as the total number of co-residents per household divided by the number of rooms, excluding kitchens, bathrooms, hallways, balconies and garage ${ }^{(35)}$. The income group classification was based on the legal minimum wage in Lebanon, approxi-

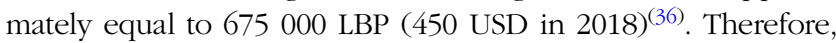
low-income household was defined as $\leq 750 \cdot 000$ LBP and highincome household as $>750 \cdot 000$ LBP (500 USD in 2018).

FI was measured at the individual level for the mother using the global Food Insecurity Experience Scale. The prevalence of moderate and severe FI in the total study population represents the Sustainable Development Goal indicator 2.1.2. The Food Insecurity Experience Scale generates two indicators, the FI prevalence rates at the moderate and severe level and at the severe level only, by computing the probability of being food insecure for each individual ${ }^{(37)}$. The probability of belonging to a food security class was extracted to be used in the analysis as such: probability of being moderately and severely food insecure and probability of being severely food insecure. Food Insecurity Experience Scale was internally validated using statistical methods based on the Rasch measurement model. The development of the score and validation process is published elsewhere $^{(38,39)}$.

\section{Anthropometric and biochemical assessment}

Mid-upper arm, waist and hip circumference were measured among mothers with light clothing using a non-elastic measuring tape (SECA 201). An average of two measurements was recorded to the nearest decimal using standardised protocols ${ }^{(40)}$. The classification of malnutrition using the mid-upper arm circumference was applied for all mothers as follows: $<23.0 \mathrm{~cm}$ for undernourished, $23.0-27.9 \mathrm{~cm}$ for normal weight and $\geq 28.0 \mathrm{~cm}$ for overweight and obese mothers ${ }^{(41-44)}$. For non-PM, the BMI was computed as the weight $(\mathrm{kg})$ divided by the height squared $\left(\mathrm{m}^{2}\right)$. BMI classification was categorised into two groups 'Underweight' (BMI $<18.5 \mathrm{~kg} / \mathrm{m}^{2}$ ), 'Normal weight' (BMI 18.5 $24.9 \mathrm{~kg} / \mathrm{m}^{2}$ ) and 'Overweight/Obese' (BMI $\left.\geq 25.0 \mathrm{~kg} / \mathrm{m}^{2}\right)^{(45)}$. $\mathrm{Hb}$ concentrations were measured using the 'HemoCue Hb301 System' by certified phlebotomists. The accuracy of the measurement was routinely ensured with the use of control solutions. A small drop of blood was collected by conducting a finger prick on mothers. Anaemia cut-offs were defined using the WHO criteria. Total anaemia was set at $\mathrm{Hb}<12.0 \mathrm{~g} / \mathrm{dl}$ for nonpregnant non-lactating and $\mathrm{LM}$ and at $\mathrm{Hb}<11.0 \mathrm{~g} / \mathrm{dl}$ for PM at sea level(46).

\section{Dietary assessment}

Data were collected on the characteristics of daily meal patterns and the dietary intake of the mother using two dietary assessment methods: (1) a 24-h dietary recall during the previous day and (2) a FFQ during the past 12 months. The 24-h dietary recall was used for the assessment of maternal dietary diversity and nutrient inadequacies, whereas the FFQ method was used to assess the percentage contribution of food groups to total energy consumption. Both dietary assessment methods were conducted by trained nutritionists using the $2 \mathrm{D}$ food portion visual ${ }^{(47)}$ and standardised reference portions to facilitate the collection of dietary data and ensure standardisation. The five-step multiple-pass 24-h dietary recall method was used to assess the dietary intake during the previous day, which included the following steps: (1) start with a quick uninterrupted listing of foods by the interviewee, (2) probe for forgotten foods list, (3) collect the time and occasion, (4) gather a comprehensive description of foods and amounts eaten in the detailed cycle and (5) end with a final probe review ${ }^{(48,49)}$. Furthermore, a ninety-four-item semiquantitative context-specific FFQ, validated among adults in Lebanon $^{(50)}$, was used to assess the dietary intake of mothers over the past 12 months. Dietary data stemming from the FFQ were combined into numerous food groups and subgroups in order to examine their contribution to the percentage energy intake (\% EI). Dietary data were analysed using the United States Department of Agriculture (USDA) database (SR 28, version: May 2016) which was complemented with single food items from local food composition tables available for specific Lebanese foods, given the similarities between Eastern Mediterranean cuisines ${ }^{(51,52)}$. Standardised recipes were added to analyse composite and traditional Lebanese and Syrian dishes. NutriSurvey 2007 software was used for the analysis of macroand micronutrients' intake from the $24-\mathrm{h}$ recalls ${ }^{(53)}$.

\section{Minimum Dietary Diversity for Women of reproductive age}

Based on the 24-h dietary recalls, maternal dietary diversity was assessed using the Minimum Dietary Diversity for Women (MDD-W) of reproductive age. Food items were aggregated into sixteen food groups as follows: (1) grains, white roots and tubers, (2) pulses (beans, peas and lentils), (3) nuts and seeds, (4) dairy products, (5) meat, poultry and fish, (6) eggs, (7) dark green leafy vegetables, (8) other vitamin A-rich fruits and vegetables, (9) other vegetables, (10) other fruits, (11) other oils and fats, (12) fried and salty foods, (13) sweet foods, (14) sugar-sweetened beverages, (15) condiments and seasonings and (16) other beverages and foods. Food items consumed in very small quantities $(<15 \mathrm{~g})$ were placed in the 'Condiments and seasonings' category. Sweetened coffees, teas and milk were placed in the 'Sweetened beverages' category. Mixed and traditional dishes (e.g. stews, soups, vegetable dishes, sandwiches) were carefully evaluated to classify main ingredients consumed in large quantities in their respective food groups and minor ingredients to other groups such as groups 11 and/or 15. Composite dishes and food items (e.g. bread, pizza, porridge, fried savoury, sweets and pastries, sweet drinks) were assigned to a single food group based on the major ingredient. Food group diversity score was 
computed by summing up the first ten MDD-W food groups consumed yesterday into a score ranging from 0 to 10 . The MDD-W indicator defined achieving MDD-W as a food group diversity score ranging from 5 to 10 and LDD as not achieving MDD $(<5 \text { food groups yesterday })^{(11)}$.

\section{Nutrient inadequacies}

Nutrient intakes stemming from the 24-h dietary recalls were used to assess the prevalence of inadequate intakes by comparing the intakes of a nutrient to the estimated average requirements (EAR) according to the Institute of Medicine. The EAR is the average daily nutrient intake level estimated to meet the requirements of half of the mothers according to their age and reproductive status groups (pregnant, lactating or non-pregnant non-lactating). The adequate intake was used in the absence of an EAR, as for dietary fibres ${ }^{(54)}$. The analysed micronutrients represent those for which the MDD-W indicator was validated against ${ }^{(11)}$. Further analysis was conducted using the criterion of two-thirds of the Dietary Reference Intakes which has been commonly used by researchers as a target ${ }^{(55)}$ and in previous national studies in Lebanon ${ }^{(56-58)}$. Dietary Reference Intakes refer to the RDA and adequate intakes for selected macroand micronutrients ${ }^{(54)}$. This criterion represents the proportion of mothers failing to meet two-thirds of the RDA or adequate intake for key macro- and micronutrients taking into account their age and reproductive status.

\section{Statistical analysis}

Data entry was completed with the support of KOBO Technology provided by Harvard Humanitarian Institute ${ }^{(59)}$. Data analysis was carried out using the Statistical Package for Social Sciences, version 27.0 (SPSS Inc.). Descriptive statistics were expressed as mean and standard deviation for continuous variables or as number of subjects and percentages $(n, \%)$ for nominal variables. Reproductive status of the mother was categorised as PM, non-pregnant LM and non-pregnant non-lactating mothers (NPNLM). Associations were investigated between the reproductive status of the mother and the MDD-W indicator and food group diversity score using $\chi^{2}$ test for categorical variables and one-way ANOVA test to compare means. Simple and multiple binary logistic regressions were used to examine the association between maternal LDD (dependent variable) and socioeconomic and household characteristics, healthcare service utilisation, anaemia, nutritional and mental health status of the mothers, daily meal patterns of mothers and nutrient inadequacies. Regression models were assessed for significance by evaluating the $R^{2}$, overall percentage and Hosmer and Lemeshow test. Crude odds were expressed as OR and adjusted OR as aOR with $95 \%$ CI. Point biserial and Pearson's correlation tests were used to examine the association between the probability of being food insecure and key variables in the study. Independent-samples $t$ test was used to examine the associations between the main food groups and subgroups from the FFQ and the low- and highincome group. Statistical significance was defined as $P$-value $<0 \cdot 05$. Correlation coefficients, tolerance, variance inflation factors and the condition index were used to measure multicollinearity on all the independent variables.

\section{Results}

A total of 433 subjects were included in the final analysis of the study, of which $17 \cdot 8 \%$ were PM, $35 \cdot 1 \%$ NPNLM and $47 \cdot 1 \%$ LM. The prevalence of moderate and severe FI in the total study population was $34 \cdot 4 \%$ (SD $0 \cdot 1$ ), whereby the prevalence of severe FI reached $12.5 \%$ (SD $0 \cdot 1)$. No significant differences were found between the prevalence of FI and the reproductive status groups of mothers (data not shown in table). Nearly two out of three Syrian refugee mothers had a LDD (63.3\%) in the present study, while only $36.7 \%$ of the mothers achieved MDD-W. On average, mothers with LDD consumed $3 \cdot 2$ (SD 0.8) food groups per day, while mothers with MDD-W consumed 5.6 (SD 0.8) food groups, with scores ranging from 1 to 9 food groups (Table 1).

As shown in Table 1, the MDD-W indicator and food groups consumed yesterday by mothers were presented according to their reproductive status and based on the 24-h dietary recalls. Overall, no significant differences in the MDD-W indicator were observed among PM, LM and NPNLM. Nearly two-thirds of the mothers consumed less than five food groups over the previous day (PM: 62.3\%, LM: 62.7\%, NPNLM: 64.5\%), with an average consumption of 4.1 (SD 1.4) food groups. Almost all mothers in the study sample consumed grains, white roots and tubers (99.5\%). Sugar-sweetened beverages (82.0\%) and oils and fats $(79.7 \%)$ were consumed by a majority and nearly half of the mothers had eaten sweets $(42 \cdot 7 \%)$ during the previous day. The consumption of fruits and vegetables was reportedly high (86.8\%); however, dark green leafy vegetables and vitamin Arich fruits and vegetables were rarely included in their diet (11.8 and $13.4 \%)$. Mothers consumed largely other vegetables $(74.8 \%)$ and moderately other fruits (39.0\%). Similarly, animal-source foods were widely consumed ( $86 \cdot 6 \%$ ), yet less than $50 \%$ ate meat, poultry and fish (44.6\%) or eggs (29.8\%) while dairy products were more prevalent in their diet (68.4\%). The lowest consumption was observed for pulses, nuts and seeds (27.3\%) among all mothers. LM consumed more dairy products and other beverages and foods than PM and NPNLM, while PM had a higher intake of eggs as compared with LM and NPNLM $(P<0 \cdot 05)$. Furthermore, even though it did not reach a statistical difference, a higher proportion of LM consumed sweets, sugarsweetened beverages, other fats and oils, as compared with PM and NPNLM.

Nutrient inadequacies of key macro- and micronutrients according to maternal LDD, based on 24-h dietary recalls, are shown in Table 2. High prevalence of inadequate intakes was found among mothers for proteins, fibres, folate, vitamins $\mathrm{B}_{12}$, $\mathrm{B}_{6}, \mathrm{C}$ and $\mathrm{A}, \mathrm{Zn}$ and $\mathrm{Ca}$ ranging from $70 \cdot 8$ to $89.6 \%$. Moreover, more than half of the mothers did not meet the EAR for riboflavin (64.7\%), Fe (59.5\%) and niacin (55.7\%). Simple regressions showed that maternal LDD was significantly associated with nutrient inadequacies ( $<$ EAR) for key vitamins, minerals and macronutrients studied. After adjusting for the energy intake of mothers, significant associations between LDD and nutritional inadequacies persisted for vitamins $\mathrm{B}_{12}, \mathrm{C}$ and $\mathrm{A}$ and riboflavin. The odds of nutrient inadequacies increased by two to three times among mothers with LDD as compared with mothers who achieved MDD-W. Further analysis displaying the proportion of mothers failing to meet two-thirds of 
Table 1. MDD-W indicator among Syrian mothers according to their reproductive status based on 24-h dietary recalls (Mean values and standard deviations)

\begin{tabular}{|c|c|c|c|c|c|c|c|c|c|}
\hline \multirow[b]{2}{*}{ Variables* ${ }^{*}$} & \multicolumn{2}{|c|}{$\begin{array}{c}\text { Pregnant } \\
\text { mothers } n 77 \\
\end{array}$} & \multicolumn{2}{|c|}{$\begin{array}{c}\text { Lactating } \\
\text { mothers } n 204\end{array}$} & \multicolumn{2}{|c|}{ NPNLM n 152} & \multicolumn{2}{|c|}{ Total $n 433$} & \multirow[b]{2}{*}{ P† } \\
\hline & $n$ & $\%$ & $n$ & $\%$ & $n$ & $\%$ & $n$ & $\%$ & \\
\hline \multicolumn{10}{|l|}{ MDD-W indicator } \\
\hline \multicolumn{10}{|l|}{ Food group diversity scoreł } \\
\hline Mean & \multirow{2}{*}{\multicolumn{2}{|c|}{$\begin{array}{c}4.17 \\
1.5\end{array}$}} & \multicolumn{2}{|c|}{4.19} & \multicolumn{2}{|c|}{4.04} & \multicolumn{2}{|c|}{$4 \cdot 13$} & 0.591 \\
\hline SD & & & & & & & & & \\
\hline \multicolumn{10}{|l|}{ MDD-W indicator§ } \\
\hline LDD (< 5 out of 10 food groups) & 48 & $62 \cdot 3$ & 128 & $62 \cdot 7$ & 98 & $64 \cdot 5$ & 274 & 63.3 & \\
\hline MDD-W ( $\geq 5$ out of 10 food groups) & 29 & 37.7 & 76 & 37.3 & 54 & 35.5 & 159 & $36 \cdot 7$ & 0.929 \\
\hline \multicolumn{10}{|l|}{ Consumption of nutrient-rich food groups } \\
\hline Pulses, nuts and seeds $\|$ & 22 & 28.6 & 52 & 25.5 & 44 & 28.9 & 118 & $27 \cdot 3$ & 0.738 \\
\hline Animal-source foods & 65 & 84.4 & 185 & $90 \cdot 7$ & 125 & $82 \cdot 2$ & 375 & $86 \cdot 6$ & 0.056 \\
\hline Fruits and vegetables ${ }^{\star *}$ & 68 & 88.3 & 171 & 83.8 & 137 & $90 \cdot 1$ & 376 & $86 \cdot 8$ & 0.201 \\
\hline \multicolumn{10}{|c|}{ MDD-W food groups consumed on the day before the interview } \\
\hline 1. Grain, white roots and tubers & 77 & $100 \cdot 0$ & 204 & $100 \cdot 0$ & 150 & 98.7 & 431 & 99.5 & 0.156 \\
\hline 2. Pulses (beans, peas and lentils) & 15 & $19 \cdot 5$ & 41 & $20 \cdot 1$ & 30 & $19 \cdot 7$ & 86 & $19 \cdot 9$ & 0.992 \\
\hline 3. Nuts and seeds & 11 & $14 \cdot 3$ & 20 & $9 \cdot 8$ & 22 & 14.5 & 53 & $12 \cdot 2$ & 0.344 \\
\hline 4. Dairy & 51 & $66 \cdot 2$ & 152 & 74.5 & 93 & $61 \cdot 2$ & 296 & $68 \cdot 4$ & 0.025 \\
\hline 5. Meat, poultry and fish & 27 & $35 \cdot 1$ & 97 & 47.5 & 69 & $45 \cdot 4$ & 193 & $44 \cdot 6$ & 0.166 \\
\hline 6. Eggs & 29 & 37.7 & 66 & 32.4 & 34 & $22 \cdot 4$ & 129 & $29 \cdot 8$ & 0.031 \\
\hline 7. Dark green leafy vegetables & 7 & $9 \cdot 1$ & 24 & $11 \cdot 8$ & 20 & $13 \cdot 2$ & 51 & $11 \cdot 8$ & 0.666 \\
\hline 8. Other vitamin A-rich fruits and vegetables & 9 & 11.7 & 27 & 13.2 & 22 & 14.5 & 58 & 13.4 & 0.839 \\
\hline 9. Other vegetables & 58 & $75 \cdot 3$ & 154 & 75.5 & 112 & 73.7 & 324 & $74 \cdot 8$ & 0.922 \\
\hline 10. Other fruits & 37 & $48 \cdot 1$ & 70 & 34.3 & 62 & $40 \cdot 8$ & 169 & $39 \cdot 0$ & 0.094 \\
\hline 11. Other oils and fats & 58 & $75 \cdot 3$ & 170 & 83.3 & 117 & $77 \cdot 0$ & 345 & $79 \cdot 7$ & 0.195 \\
\hline 12. Fried and salty foods & 8 & $10 \cdot 4$ & 14 & 6.9 & 12 & 7.9 & 34 & 7.9 & 0.618 \\
\hline 13. Sweet foods & 28 & 36.4 & 95 & 46.6 & 62 & $40 \cdot 8$ & 185 & $42 \cdot 7$ & 0.254 \\
\hline 14. Sugar-sweetened beverages & 61 & $79 \cdot 2$ & 171 & 83.8 & 123 & $80 \cdot 9$ & 355 & $82 \cdot 0$ & 0.612 \\
\hline 15. Condiments and seasonings & 67 & 87.0 & 170 & 83.3 & 131 & $86 \cdot 2$ & 368 & $85 \cdot 0$ & 0.652 \\
\hline 16. Other beverages and foods & 22 & 28.6 & 98 & 48.0 & 67 & $44 \cdot 1$ & 187 & $43 \cdot 2$ & 0.013 \\
\hline
\end{tabular}

MDD-W, Minimum Dietary Diversity for Women; NPNLM, non-pregnant non-lactating mothers; LDD, low dietary diversity.

* Categorical variables are expressed as $n(\%)$ and continuous variables are expressed as mean values and standard deviations.

** The variable 'fruits and vegetables' is the sum of food groups $7,8,9$ and 10 .

† Significantly different at $P$-value $<0.05 ; P$-value was derived using one-way ANOVA test for continuous variables and $\chi^{2}$ analysis for categorical variables.

¥ Food group diversity score is the sum of the first ten MDD-W food groups consumed yesterday.

$\S$ The MDD-W indicator defined LDD as a food group diversity score $<5$ and MDD-W as $\geq 5$ out of ten food groups.

॥ The variable 'pulses, nuts and seeds' is the sum of food groups 2 and 3 .

IT The variable 'animal-source foods' is the sum of food groups 4,5 and 6 .

the RDA or adequate intake for key macro- and micronutrients is shown in online Supplementary Table S1. Findings showed a high prevalence of nutrient intake below two-thirds of Dietary Reference Intake for the majority of the studied macro- and micronutrients. After adjusting for energy, significant associations remained between LDD and intake below two-thirds of Dietary Reference Intake for a wide array of vitamins and minerals as well as protein and dietary fibres.

Key determinants of LDD among Syrian mothers of children under 5, including socio-economic and household characteristics, healthcare service utilisation, maternal daily meal pattern, anaemia and nutritional status, are presented in Table 3. Simple logistic regression analysis showed that LDD among mothers was significantly associated with mother's education, father's employment status, household monthly income, UNHCR registration status as a refugee, being a beneficiary of WFP food assistance, crowding index score, mother's use of micronutrient supplements, number of antenatal care visits during the previous pregnancy and sources of health and nutrition messages from healthcare professionals $(P<0.05)$. Using the multiple logistic regression model, mothers in households with a monthly income $\leq 750000$ LBP had a two times higher odds of having $\mathrm{LDD}(\mathrm{aOR}=2 \cdot 09$; $95 \% \mathrm{CI} 1 \cdot 19,3 \cdot 67)$ than those in households with a higher income. In addition, LDD was significantly associated with higher odds of being registered as a refugee with the UNHCR ( $\mathrm{aOR}=2 \cdot 13 ; 95 \% \mathrm{CI} 1 \cdot 07,4 \cdot 24)$, receiving WFP food assistance (e-vouchers) $(\mathrm{aOR}=3.70 ; 95 \% \mathrm{CI} 1.09,12.53)$ and $\mathrm{a}$ higher crowding index score $(\mathrm{aOR}=1 \cdot 32 ; 95 \% \mathrm{CI} 1 \cdot 07,1 \cdot 64)$. On the other hand, mothers with LDD were less likely to use micronutrient supplements (aOR $=0.49 ; 95 \%$ CI 0.27, 0.88). LDD was also inversely associated with receiving health and nutrition messages from a community healthcare worker and from multiple healthcare professionals $(\mathrm{aOR}=0.27 ; 95 \% \mathrm{CI} 0 \cdot 08,0.84$ and $\mathrm{aOR}=0 \cdot 04 ; 95 \% \mathrm{CI} 0 \cdot 00,0 \cdot 43$, respectively) as compared with mothers who did not receive any messages. Mother's education, father's employment and number of antenatal care visits were no longer statistically significantly associated with LDD after adjusting for socio-economic variables. Mothers consuming a daily breakfast and a higher number of snacks per day were significantly less likely to have LDD $(P<0 \cdot 05)$. No significant associations were found between maternal dietary diversity, anaemia and nutritional status of the mother. 
Table 2. Nutrient inadequacy of key macro- and micronutrients according to maternal low dietary diversity based on 24 -hour dietary recalls

\begin{tabular}{|c|c|c|c|c|c|c|c|c|}
\hline \multirow[b]{2}{*}{ Variables ${ }^{\star *}$} & \multirow[b]{2}{*}{ EAR† } & \multirow{2}{*}{$\begin{array}{c}\text { MDD-W } \\
n=159\end{array}$} & \multirow{2}{*}{$\begin{array}{c}\text { LDD } \\
n=274\end{array}$} & \multirow{2}{*}{$\begin{array}{c}\text { Total } \\
(N=433)\end{array}$} & \multicolumn{4}{|c|}{ Maternal Low Dietary Diversity } \\
\hline & & & & & OR $\ddagger(95 \% \mathrm{Cl})$ & $P$ & aOR§ $(95 \% \mathrm{Cl})$ & $P$ \\
\hline \multicolumn{9}{|l|}{ Macronutrient intake } \\
\hline Protein (g/kg/d) & $0.66-1.05$ & & & & & & & \\
\hline$\geq \mathrm{EAR}$ & & $58(36 \cdot 7)$ & $49(17.9)$ & $107(24 \cdot 8)$ & 1.0 & & 1.0 & \\
\hline$<\mathrm{EAR}$ & & $100(63.3)$ & $225(82 \cdot 1)$ & $325(75 \cdot 2)$ & $2.66(1.70-4 \cdot 17)$ & 0.000 & $1.38(0.82-2 \cdot 32)$ & 0.229 \\
\hline$\geq \mathrm{EAR}$ & & $132(83.0)$ & $169(61.7)$ & $301(69.5)$ & 1.0 & & 1.0 & \\
\hline$<$ EAR & & $27(17.0)$ & $105(38.3)$ & $132(30.5)$ & $3.04(1.88-4.91)$ & 0.000 & $1.40(0.78-2.51)$ & 0.260 \\
\hline Fibers (g/d) & $25-29^{*}$ & & & & & & & \\
\hline$\geq \mathrm{Al}$ & & $24(15 \cdot 2)$ & $21(7 \cdot 7)$ & $45(10 \cdot 4)$ & $1 \cdot 0$ & & $1 \cdot 0$ & \\
\hline$<\mathrm{Al}$ & & $134(84.8)$ & $253(92 \cdot 3)$ & $387(89.6)$ & $2.16(1.16-4.02)$ & 0.015 & $0.74(0.35-1.56)$ & 0.430 \\
\hline \multicolumn{9}{|l|}{ Micronutrient intake } \\
\hline Iron (mg/d) & $6 \cdot 5-23$ & & & & & & & \\
\hline Folate $(\mu \mathrm{g} / \mathrm{d})$ & $320-520$ & & & & & & & \\
\hline$\geq E A R$ & & $28(17 \cdot 7)$ & $18(6 \cdot 6)$ & $46(10.6)$ & 1.0 & & 1.0 & \\
\hline$<$ EAR & & $130(82.3)$ & $256(93.4)$ & $386(89.4)$ & $3.06(1.63-5.74)$ & 0.000 & $1.57(0.79-3.13)$ & 0.200 \\
\hline Vitamin B12 $(\mu \mathrm{g} / \mathrm{d})$ & $2 \cdot 0-2 \cdot 4$ & & & & & & & \\
\hline$\geq$ EAR & & $42(26 \cdot 4)$ & $22(8.0)$ & $64(14.8)$ & 1.0 & & 1.0 & \\
\hline$<$ EAR & & $117(73.6)$ & $252(92 \cdot 0)$ & $369(85.2)$ & $4 \cdot 11(2 \cdot 35-7 \cdot 20)$ & 0.000 & $3.08(1.70-5.56)$ & 0.000 \\
\hline Vitamin C (mg/d) & $56-100$ & & & & & & & \\
\hline$\geq \mathrm{EAR}$ & & $68(43.0)$ & $58(21 \cdot 2)$ & $126(29 \cdot 2)$ & 1.0 & & 1.0 & \\
\hline$<$ EAR & & $90(57.0)$ & $216(78.8)$ & $306(70.8)$ & $2.81(1.83-4.32)$ & 0.000 & $1.96(1.24-3.11)$ & 0.004 \\
\hline Vitamin $A(\mu \mathrm{g} / \mathrm{d})$ & 485-900 & & & & & & & \\
\hline$\geq \mathrm{EAR}$ & & $34(21.5)$ & $23(8.4)$ & $57(13 \cdot 2)$ & 1.0 & & 1.0 & \\
\hline$<$ EAR & & $124(78.5)$ & $251(91.6)$ & $375(86.8)$ & $2.99(1.69-5.30)$ & 0.000 & $2.33(1.27-4.26)$ & 0.006 \\
\hline Thiamin (mg/d) & $0 \cdot 9-1 \cdot 2$ & & & & & & & \\
\hline$\geq \mathrm{EAR}$ & & $102(64 \cdot 2)$ & $135(49 \cdot 3)$ & $237(54 \cdot 7)$ & 1.0 & & 1.0 & \\
\hline$<$ EAR & & $57(35 \cdot 8)$ & $139(50 \cdot 7)$ & $196(45 \cdot 3)$ & $1.84(1.23-2 \cdot 75)$ & 0.003 & $0.83(0.51-1.37)$ & 0.471 \\
\hline Riboflavin (mg/d) & $0.9-1.3$ & & & & & & & \\
\hline$\geq$ EAR & & $91(57 \cdot 2)$ & $62(22 \cdot 6)$ & $153(35 \cdot 3)$ & 1.0 & & 1.0 & \\
\hline$\geq \mathrm{EAR}$ & & $96(60.4)$ & $96(35.0)$ & $192(44 \cdot 3)$ & 1.0 & & 1.0 & \\
\hline$<$ EAR & & $63(39.6)$ & $178(65.0)$ & $241(55 \cdot 7)$ & $2.82(1.89-4.23)$ & 0.000 & $1.44(0.87-2.38)$ & 0.157 \\
\hline Vitamin B6 (mg/d) & $1.0-1.7$ & & & & & & & \\
\hline$\geq \mathrm{EAR}$ & & $40(25 \cdot 3)$ & $23(8.4)$ & $63(14.6)$ & 1.0 & & 1.0 & \\
\hline$<$ EAR & & $118(74.7)$ & $251(91.6)$ & $369(85.4)$ & $3.70(2.12-6.46)$ & 0.000 & $1.75(0.92-3.32)$ & 0.086 \\
\hline Zinc (mg/d) & $8 \cdot 5-10 \cdot 9$ & & & & & & & \\
\hline$\geq \mathrm{EAR}$ & & $23(27 \cdot 2)$ & $27(9 \cdot 9)$ & $70(16 \cdot 2)$ & 1.0 & & 1.0 & \\
\hline$<\mathrm{EAR}$ & & $115(72.8)$ & $247(90 \cdot 1)$ & $362(83.8)$ & $3.42(2.01-5.81)$ & 0.000 & $1.66(0.90-3.05)$ & 0.105 \\
\hline Calcium (mg/d) & $800-1100$ & & & & & & & \\
\hline$\geq \mathrm{EAR}$ & & $27(17 \cdot 1)$ & $23(8.4)$ & $50(11.6)$ & 1.0 & & 1.0 & \\
\hline$<$ EAR & & $131(82.9)$ & $251(91.6)$ & $382(88.4)$ & $2.25(1.24-4.08)$ & 0.008 & $1.06(0.54-2.08)$ & 0.861 \\
\hline
\end{tabular}

LDD, low Dietary Diversity; EAR, estimated average requirements; MDD-W, Minimum Dietary Diversity for Women; aOR, adjusted OR; AI, adequate intake.

Al is presented in ordinary types followed by an asterisk $\left(^{*}\right)^{(54)}$.

${ }_{* \star}$ Categorical variables are expressed as $\mathrm{n}(\%)$.

t EAR is the average daily nutrient intake level estimated to meet the requirements of half of the mothers according to their age and reproductive status groups.

$\ddagger$ OR of the dependent variable (MDD-W vs. LDD) are presented with $95 \% \mathrm{Cl}$ using simple logistic regression. aOR are presented with $95 \% \mathrm{Cl}$ using multiple logistic regression analysis.

$\S$ Model 1: adjusted for total energy intake.

Statistical significance was defined as $P$-value $<0.05$

The mental health status of mothers according to maternal LDD and its associations are displayed in Table 4 . The prevalence of PTSD was $13.2 \%, 16 \cdot 2 \%$ for mild depression, $11 \cdot 1 \%$ for moderate depression and $9.9 \%$ for severe depression. In total, $27 \cdot 1 \%$ of the mothers suffered from PTSD and/or moderate/severe depression. Simple logistic regressions showed that mothers with PTSD, mild depression or severe depression had significantly higher odds of LDD $(P<0 \cdot 05)$. Similarly, mothers with PTSD and/or moderate/severe depression combined had
1.6 times higher odds of LDD as compared with those not suffering from PTSD or depression. However, these associations did not remain significant after adjusting for socio-economic characteristics and maternal anaemia. Additional analysis showed that mothers with a higher crowding index had 1.2 times higher odds of suffering from PTSD and/or moderate/severe depression ( $\mathrm{aOR}=1 \cdot 24,95 \% \mathrm{CI} 1 \cdot 01,1 \cdot 52)$. Furthermore, a higher proportion of mothers suffering from the flu were found to have a crowding index higher than 2 as compared with those 
Table 3. Key determinants of maternal low dietary diversity among Syrian mothers

\begin{tabular}{|c|c|c|c|c|c|c|}
\hline \multirow[b]{2}{*}{ Variables* $^{*}$} & \multirow{2}{*}{$\begin{array}{c}\text { MDD-W } \\
n=159 \\
\end{array}$} & \multirow{2}{*}{$\begin{array}{c}\text { LDD } \\
n=274\end{array}$} & \multicolumn{4}{|c|}{ Maternal Low Dietary Diversity } \\
\hline & & & $\mathrm{OR}^{\star \star}(95 \% \mathrm{Cl})$ & $P$ & $\mathrm{aOR} \dagger(95 \% \mathrm{Cl})$ & $P$ \\
\hline \multicolumn{7}{|l|}{ Socio-economic characteristics } \\
\hline \multicolumn{7}{|l|}{ Mother's education level } \\
\hline No schooling/lliterate & $20(12 \cdot 7)$ & $50(18.5)$ & 1.0 & & 1.0 & \\
\hline Primary, Intermediate school & $84(53.2)$ & $165(60.9)$ & $0.78(0.44-1.40)$ & 0.416 & $0.87(0.39-1.94)$ & 0.727 \\
\hline Secondary school and higher & $54(34.2)$ & $56(20.7)$ & $0.42(0.22-0.79)$ & 0.007 & $0.97(0.38-2.45)$ & 0.943 \\
\hline \multicolumn{7}{|l|}{ Father's employment status } \\
\hline Full-time job/Self-employed & $92(58.6)$ & $122(46 \cdot 9)$ & $1 \cdot 0$ & & $1 \cdot 0$ & \\
\hline No job/Part-time job & $65(41.4)$ & $138(53 \cdot 1)$ & $1.60(1.07-2.39)$ & 0.021 & $1.50(0.89-2.55)$ & 0.132 \\
\hline \multicolumn{7}{|l|}{ Monthly household income } \\
\hline$\leq 750,000$ LBP & $79(53.4)$ & $190(72 \cdot 2)$ & $2 \cdot 27(1.49-3.46)$ & 0.000 & $2.09(1.19-3.67)$ & 0.010 \\
\hline$>750,000 \mathrm{LBP}$ & $69(46.6)$ & $73(27.8)$ & 1.0 & & $1 \cdot 0$ & \\
\hline \multicolumn{7}{|l|}{ UNHCR refugee registration status } \\
\hline No & $39(25.3)$ & $37(13.7)$ & 1.0 & & 1.0 & \\
\hline Yes & $115(74.7)$ & $234(86 \cdot 3)$ & $2.14(1.30-3.54)$ & 0.003 & $2.13(1.07-4.24)$ & 0.031 \\
\hline \multicolumn{7}{|l|}{ Receiving WFP food assistance (e-vouchers) } \\
\hline No & $148(96.7)$ & $244(90.4)$ & 1.0 & & 1.0 & \\
\hline Yes & $5(3.3)$ & $26(9.6)$ & $3.15(1.18-8.39)$ & 0.021 & $3.70(1.09-12.53) \ddagger$ & 0.035 \\
\hline Crowding index score (mean $\pm \mathrm{SD}$ ) & $3.27(1.2)$ & $3.95(1.8)$ & $1.34(1.17-1.55)$ & 0.000 & $1.32(1.07-1.64)$ & 0.031 \\
\hline \multicolumn{7}{|l|}{ Healthcare service utilization } \\
\hline \multicolumn{7}{|l|}{ Mother's use of micronutrients supplements } \\
\hline No & $99(63.1)$ & $200(73.8)$ & 1.0 & & 1.0 & \\
\hline Yes & $58(36.9)$ & $71(26 \cdot 2)$ & $0.61(0.39-0.93)$ & 0.020 & $0.49(0.27-0.88)$ & 0.018 \\
\hline \multicolumn{7}{|l|}{ Number of antenatal care visits } \\
\hline 0 times & $26(16.7)$ & $45(16 \cdot 6)$ & $1.21(0.70-2.08)$ & 0.491 & $0.85(0.42-1.73)$ & 0.666 \\
\hline 1 to 3 times & $21(13.5)$ & $70(25.8)$ & $2.33(1.35-4.02)$ & 0.002 & $2.01(1.00-4.02)$ & 0.050 \\
\hline$\geq 4$ times & $109(69.9)$ & $156(57.6)$ & 1.0 & & 1.0 & \\
\hline \multicolumn{7}{|l|}{$\begin{array}{l}\text { Sources of health and nutrition messages from } \\
\text { healthcare professionals only }\end{array}$} \\
\hline No healthcare professionals & $105(66 \cdot 0)$ & $203(74.4)$ & $1 \cdot 0$ & & $1 \cdot 0$ & \\
\hline One healthcare professional§ & $36(22 \cdot 6)$ & $57(20.9)$ & $0.82(0.51-1.32)$ & 0.414 & $0.72(0.38-1.35)$ & 0.309 \\
\hline Community healthcare worker & $9(5 \cdot 7)$ & $9(3.3)$ & $0.52(0.20-1.34)$ & 0.175 & $0.27(0.08-0.84)$ & 0.024 \\
\hline Multiple healthcare professionals & $9(5 \cdot 7)$ & $4(1.5)$ & $0.23(0.07-0.76)$ & 0.016 & $0.04(0.00-0.43)$ & 0.008 \\
\hline \multicolumn{7}{|l|}{ Maternal daily meal pattern } \\
\hline \multicolumn{7}{|l|}{ Have breakfast every day } \\
\hline No & $37(23.9)$ & $91(33 \cdot 3)$ & 1.0 & & 1.0 & \\
\hline Yes & $118(76 \cdot 1)$ & $182(66 \cdot 7)$ & $0.63(0.40-0.98)$ & 0.041 & $0.55(0.31-0.99)$ & 0.045 \\
\hline Number of main meals per day (mean $\pm \mathrm{SD}$ ) & $2.19(0.6)$ & $2.08(0.6)$ & $0.73(0.53-1.02)$ & 0.067 & $0.54(0.35-0.85)$ & 0.008 \\
\hline Number of snacks per day (mean $\pm \mathrm{SD}$ ) & $1.54(1.0)$ & $1.19(1.0)$ & $0.72(0.59-0.88)$ & 0.001 & $0.76(0.59-0.99)$ & 0.038 \\
\hline \multicolumn{7}{|l|}{ Anemia and nutritional status of all mothers } \\
\hline \multicolumn{7}{|l|}{ Maternal anemia status } \\
\hline Not anemic & $122(77.7)$ & $214(78.7)$ & 1.0 & & 1.0 & \\
\hline Anemic & $35(22.3)$ & $58(21 \cdot 3)$ & $0.94(0.59-1.52)$ & 0.814 & $1.17(0.61-2.23)$ & 0.643 \\
\hline \multicolumn{7}{|l|}{ Classification of malnutrition using MUAC } \\
\hline Normal weight $(23.0-27.9 \mathrm{~cm})$ & $47(29.6)$ & $99(36.4)$ & $1 \cdot 0$ & & 1.0 & \\
\hline Undernourished $(<23.0 \mathrm{~cm})$ & $9(5 \cdot 7)$ & $11(4 \cdot 0)$ & $0.58(0.22-1.50)$ & 0.260 & $0.32(0.10-1.03)$ & 0.056 \\
\hline Overweight/Obese $(\geq 28.0 \mathrm{~cm})$ & $103(64.8)$ & $162(59.6)$ & $0.75(0.49-1.14)$ & 0.179 & $0.82(0.46-1.46)$ & 0.503 \\
\hline Nutritional status of non-pregnant mothers $\|$ & $n=130$ & $n=226$ & $\mathrm{OR}^{\star *}(95 \% \mathrm{Cl})$ & & aORף $(95 \% \mathrm{Cl})$ & \\
\hline \multicolumn{7}{|l|}{$\mathrm{BMI}$ for the mother $\left(\mathrm{kg} / \mathrm{m}^{2}\right)$} \\
\hline Normal weight $(18.5-24.9)$ & $39(30.0)$ & $92(40.7)$ & 1.0 & & 1.0 & \\
\hline Underweight $(<18.5)$ & $4(3.1)$ & $5(2 \cdot 2)$ & $0.53(0.14-2.08)$ & 0.363 & $0.58(0.12-2.76)$ & 0.492 \\
\hline Overweight/Obese $(\geq 25.0)$ & $87(66.9)$ & $129(57.1)$ & $0.63(0.40-0.99)$ & 0.049 & $0.69(0.37-1.26)$ & 0.226 \\
\hline
\end{tabular}

LDD, low dietary diversity ( $<5$ out of 10 food groups); MDD-W, Minimum Dietary Diversity for Women ( $>5$ out of 10 food groups); WFP, World Food Programme; aOR, adjusted OR; PM, pregnant mothers.

* Categorical variables are expressed as $\mathrm{n}(\%)$ and continuous variables are expressed as mean $\pm \mathrm{SD}$. Lack of corresponding sum of frequencies with total sample size is due to missing data.

** OR of the dependent variable (MDD-W vs. LDD) are presented with $95 \% \mathrm{Cl}$ using simple logistic regression.

† aOR are presented with $95 \% \mathrm{Cl}$ using multiple logistic regression analysis. Model 2: adjusted for age of the mother and child, sex of the child, reproductive status of the mother, marital status, education and employment status of the parents, household monthly income, crowding index, UNHCR registration status, perception of safety, receiving cash or food assistance, reliance on savings, household type, head of household, total number of under-five children, health insurance coverage, healthcare type, sources of health messages, the probability of being moderately and severely food insecure, and maternal anemia.

$\ddagger$ Model 3: Model 2 without receiving cash or food assistance.

$\S$ Healthcare professionals included physicians, nurses, dietitians, and pharmacists.

|| Non-PM include lactating mothers and non-pregnant non-lactating mothers.

I) Model 4: Model 2 without the age/marital status/physiological status of the mother.

Statistical significance was defined as $P$-value $<0.05$ 
Table 4. Mental health status of Syrian mothers by maternal low dietary diversity and its associations

\begin{tabular}{|c|c|c|c|c|c|c|c|}
\hline \multirow{2}{*}{$\frac{\text { Variables* }^{*}}{\text { Mental health status of the mother }}$} & \multirow{2}{*}{$\frac{\text { MDD-W }}{n=159}$} & \multirow{2}{*}{$\begin{array}{l}\text { LDD } \\
n=274\end{array}$} & \multirow{2}{*}{$\begin{array}{l}\text { Total }^{* *} \\
N=433\end{array}$} & \multicolumn{4}{|c|}{ Maternal Low Dietary Diversity } \\
\hline & & & & $\mathrm{OR} \dagger(95 \% \mathrm{Cl})$ & $P$ & aOR $\ddagger(95 \% \mathrm{Cl})$ & $P$ \\
\hline \multicolumn{8}{|l|}{ Current state of PTSD } \\
\hline No & $146(91.8)$ & $229(83.9)$ & $375(86 \cdot 8)$ & 1.0 & & 1.0 & \\
\hline Yes & $13(8 \cdot 3)$ & $44(16 \cdot 1)$ & $57(13 \cdot 2)$ & $2 \cdot 16(1 \cdot 12-4 \cdot 14)$ & 0.021 & $1.47(0.60-3.62)$ & 0.405 \\
\hline \multicolumn{8}{|l|}{ Depression screening (PHQ-9) } \\
\hline Minimal symptoms $(<5)$ & $108(71 \cdot 1)$ & $151(57.9)$ & $259(62 \cdot 7)$ & 1.0 & & $1 \cdot 0$ & \\
\hline Moderate depression (10 to 14$)$ & $17(11 \cdot 2)$ & $29(11 \cdot 1)$ & $46(11 \cdot 1)$ & $1.22(0.64-2.33)$ & 0.547 & $0.89(0.35-2.29)$ & 0.814 \\
\hline Severe depression $(\geq 15)$ & $10(6 \cdot 6)$ & $31(11.9)$ & $41(9.9)$ & $2.22(1.04-4.71)$ & 0.039 & $1.62(0.61-4.35)$ & 0.334 \\
\hline \multicolumn{8}{|l|}{ Mental health status§ } \\
\hline No PTSD or mod./sev. depression & $125(78.6)$ & $190(69 \cdot 6)$ & $315(72.9)$ & 1.0 & & $1 \cdot 0$ & \\
\hline PTSD and/or mod./sev. depression & $34(21.4)$ & $803(30.4)$ & $117(27 \cdot 1)$ & $1.61(1.02-2.54)$ & 0.043 & $1.31(0.70-2.47)$ & 0.404 \\
\hline
\end{tabular}

MDD-W, Minimum Dietary Diversity for Women; LDD, low dietary diversity; aOR, adjusted OR; PTSD, post-traumatic stress disorder; PHQ-9, Patient Health Questionnaire-9.

${ }^{*}$ Categorical variables are expressed as $\mathrm{n}(\%)$.

** Lack of corresponding sum of frequencies with total sample size is due to missing data.

† OR of the dependent variable (MDD-W vs. LDD) are presented with $95 \% \mathrm{Cl}$ using simple logistic regression.

$\ddagger$ aOR are presented with $95 \% \mathrm{Cl}$ using multiple logistic regression analysis. Model 2: adjusted for age of the mother and child, sex of the child, reproductive status of the mother, marital status, education and employment status of the parents, household monthly income, crowding index, UNHCR registration status, perception of safety, receiving cash or food assistance, reliance on savings, household type, head of household, total number of under-five children, health insurance coverage, healthcare type, sources of health messages, the probability of being moderately and severely food insecure, and maternal anemia.

$\S$ Moderate/severe (mod./sev.) depression was defined as PHQ-9 score $\geq 10^{(32)}$.

Statistical significance was defined as $P$-value $<0.05$.

without the flu $(97 \cdot 4 \% v \cdot 81 \cdot 3 \%, P<0 \cdot 05)$ (data not included in table).

Table 5 displays correlations analyses between FI and dietary indicators, mental health, anaemia, nutritional status and socioeconomic characteristics. A moderately strong significant negative correlation was found between the food group diversity score and the probability of being moderately and severely food insecure $(r=-0.283 ; P<0.000)$ as well as severely food insecure $(r=-0.251 ; P<0.000)$. Furthermore, the number of meals and snacks consumed per day was also negatively correlated with FI. As for the mental health indicators, suffering from PTSD, depression and PTSD and/or moderate/severe depression were significantly correlated with the probabilities of being food insecure $(P<0 \cdot 000)$. The strongest correlation was found between the depression screening and the probability of being moderately and severely food insecure $(r=-0.353 ; \quad P<0.000)$. Noticeable significant correlations were also found between the probability of being food insecure and the household monthly income level, employment status of the father and the crowding index. Very weak correlations were found with the education level and employment status of the mother, number of children under the age of 5 years, the reliance on savings and subsidies, registration status as refugee, head of the household and receiving health messages from healthcare professionals only. No associations were found between the probability of being food insecure and anaemia, nutritional status of the mother and the mother's use of micronutrient supplements. Further analysis showed that receiving WFP food assistance was significantly associated with a higher mean crowding index and a higher number of children aged under 5 in the household compared with not receiving WFP food assistance ( 4.7 (SD 1.8) $v$. $3.6(\mathrm{sD} 1.6)$ and $4.5(\mathrm{sD} 1.6) v .2 .5$ (SD 1.4), respectively; $P<0.000)$ (data not shown in table).

Food groups consumption of mothers based on dietary data from the FFQ according to the household monthly income is shown in Table 6. Overall, grains and starchy staples constituted the highest share of the total energy intake reaching $45.2 \%$ EI (sD 16.3) followed by $15.7 \%$ EI (SD 0.6) from fats and oils and $7.8 \% \mathrm{EI}$ (SD 6.3) from sweets and sugar derivatives. On average, dairy products, pulses, nuts, seeds and fruits contributed from $3 \cdot 2$ to $5.8 \%$ to energy consumption, while very low contributions were observed from eggs and flesh foods (2.0\% EI (SD 2.2) and $2.3 \%$ EI (SD $2 \cdot 3$ ), respectively). The lowest contribution to energy consumption was noted from the vegetables group. Other food groups such as mixed dishes, pizzas and pies, salty snacks, beverages and condiments and pickles also had a limited share in the total energy intake of about $1.7 \%$ EI to $2.7 \%$ EI.

Mothers from the low-income households had a significantly higher contribution to energy consumption from grains and starchy staples when compared with those from the highincome households, such as bread (26.5 (SD 14.7) \% EI $v .22 \cdot 2$ (sD 11.7$) \% \mathrm{EI}, P<0.05)$ and rice $(6 \cdot 2$ (sD 5.4$) \% \mathrm{EI} v .5 \cdot 1$ (sD $3.8) \% \mathrm{EI}, P<0.05)$. In contrast, the contribution to energy consumption from highly nutritious food groups was significantly lower among mothers from low-income households than those from high-income households, including fruits and fresh fruits, cheese, olive oil and flesh foods, in particular beef, lamb, organ meat and poultry $(P<0 \cdot 05)$. On the other hand, it was observed that the high-income households had a significantly higher contribution to energy consumption from sweets and sugar derivatives, especially chocolate and candies, as compared with the low-income households $(P<0.05)$. Tea consumption contributed significantly more to the dietary intake of the low-income households, as compared with high-income households $(P<0.05)$. No significant differences between the income groups were observed for vegetables, processed meat, fish, eggs, pulses, nuts, seeds, milk, yogurt, pizzas and pies, other vegetables oils and fats, salty snacks, condiments, pickles, sugar-sweetened beverages and other drinks (Table 6). 
Table 5. Correlations between food insecurity and dietary, health and socio-economic characteristics of Syrian mothers

\begin{tabular}{|c|c|c|c|c|}
\hline & \multicolumn{2}{|c|}{$\begin{array}{l}\text { Probability of being moderately } \\
\text { and severely food insecure }\end{array}$} & \multicolumn{2}{|c|}{$\begin{array}{c}\text { Probability of being severely food } \\
\text { insecure }\end{array}$} \\
\hline & Correlation coefficient $(r) \dagger$ & $P$ & Correlation coefficient $(r) \dagger$ & $P$ \\
\hline \multicolumn{5}{|l|}{ Dietary diversity and meal patterns } \\
\hline Food group diversity score ${ }^{*}(n$ 427) & -0.283 & 0.000 & -0.251 & 0.000 \\
\hline Number of main meals per day* $(n 425)$ & -0.105 & 0.042 & -0.104 & 0.032 \\
\hline Number of snacks per day* $(n 411)$ & -0.143 & 0.004 & -0.103 & 0.038 \\
\hline \multicolumn{5}{|l|}{ Mental health indicators $\ddagger$} \\
\hline Current state of PTSD ( $n$ 426) & 0.214 & 0.000 & 0.197 & 0.000 \\
\hline Depression screening (PHQ-9 $\geq 10)(n 407)$ & 0.353 & 0.000 & 0.175 & 0.000 \\
\hline Mental health status ( $n$ 426) & 0.247 & 0.000 & 0.190 & 0.000 \\
\hline \multicolumn{5}{|l|}{ Anaemia and nutritional status§ } \\
\hline Anaemia status ( $n$ 423) & 0.035 & 0.473 & -0.010 & 0.837 \\
\hline Malnutrition using MUAC ( $n$ 425) & 0.062 & 0.201 & 0.006 & 0.902 \\
\hline BMI ( $n$ 351) & -0.005 & 0.920 & -0.015 & 0.776 \\
\hline \multicolumn{5}{|l|}{ Socio-economic and household characteristics $\|$} \\
\hline Crowding index score ${ }^{*}(n$ 423) & 0.263 & 0.000 & 0.167 & 0.001 \\
\hline Monthly household income ( $n$ 406) & -0.242 & 0.000 & -0.165 & 0.001 \\
\hline Father's employment status ( $n$ 411) & -0.208 & 0.000 & -0.138 & 0.005 \\
\hline Mother's education level ( $n$ 423) & -0.179 & 0.000 & -0.063 & 0.196 \\
\hline Number of children $<5$ years* $(n 427)$ & 0.175 & 0.000 & 0.085 & 0.078 \\
\hline Reliance on savings or subsidies ( $n$ 426) & 0.144 & 0.003 & 0.158 & 0.001 \\
\hline UNHCR refugee registration status ( $n$ 419) & 0.141 & 0.004 & 0.067 & 0.170 \\
\hline Head of the household ( $n$ 420) & 0.119 & 0.015 & 0.133 & 0.006 \\
\hline Mother's employment status ( $n$ 420) & 0.085 & 0.083 & 0.113 & 0.021 \\
\hline \multicolumn{5}{|l|}{ Healthcare service utilisationๆ } \\
\hline Sources of health messages from healthcare professionals only ( $n$ 426) & -0.105 & 0.031 & -0.111 & 0.022 \\
\hline Mother's use of micronutrient supplements ( $n$ 422) & -0.073 & 0.133 & -0.030 & 0.534 \\
\hline
\end{tabular}

PTSD, post-traumatic stress disorder; PHQ-9, Patient Health Questionnaire-9; MUAC, mid-upper arm circumference.

* Indicate continuous variables.

$\dagger$ Point biserial correlation test was used for continuous and dichotomous variables. Pearson's correlation test was used for continuous variable. The strength of correlation is interpreted as follows: up to 02 (very weak), up to 05 (weak), up to 07 (moderate) and up to 09 (high). Significantly different at $P$-values $<0.05$.

$\ddagger$ Current state of PTSD (no $v$. yes), depression screening (minimal or mild depression (PHQ-9<10) $v$. moderate or severe depression (PHQ-9 $\geq 10)^{(32)}$ ), mental health status (no PTSD and/or moderate/severe depression $v$. PTSD and/or moderate/severe depression).

$\S$ Anaemia status (no $v$. yes), classification of malnutrition using MUAC (undernourished/normal weight $v$. overweight/obese), BMI (underweight/normal weight $v$. overweight/obese).

$\|$ Monthly household income ( $\leq 750000$ LBP $v .>750000$ LBP), father's employment status (full-time job $v$. no job/part-time job), mother's education level (intermediate school or lower $v$. secondary school or higher), reliance on savings or subsidies (no $v$. yes), UNHCR refugee registration status (no $v$. yes), head of the household (father/family-in-law $v$. mother/both parents), mother's employment status (no paid job/housewife $v$. paid job).

ๆ Sources of health messages from healthcare professionals only (no $v$. yes), mother's use of micronutrient supplements (no $v$. yes).

\section{Discussion}

The present study aimed to assess and examine the associations between maternal FI and dietary diversity, and mental health, anaemia and nutritional status of Syrian refugee mothers living in Greater Beirut, Lebanon. The study's main findings showed that one out of three mothers was moderately to severely food insecure $(34.4 \%$ ), with $12.5 \%$ being severely food insecure, and almost two-thirds of the mothers had a LDD (63.3\%). A large body of literature recorded a wide range of FI and LDD prevalence among women ${ }^{(60-67)}$ and refugees ${ }^{(68,69)}$ in low- and middle-income countries. Rates of FI in our study were close to those among registered Syrian refugee households in 2018-2019 across Lebanon, with one of the highest recorded in Mount Lebanon, as reported by the WFP and $\mathrm{UNHCR}^{(26,70)}$. However, higher rates of moderate to severe FI were previously found among urban Lebanese households in Greater Beirut as well as households of Palestinian and Iraqi refugees in Lebanon-(42, 61 and $80 \%$, respectively $)^{(56,71,72)}$. Lower FI rates reported in the present study may be attributed to the extensive humanitarian assistance targeting primarily Syrian refugees across Lebanon since the start of the war in 2011 and the large displacement of refugees from Syria ${ }^{(70)}$.
The prevalence of LDD in our study was found to be comparable to those from low- and middle-income countries such as mothers in Lebanon $(61.8 \%)^{(73)}$ and women refugees in Algeria $(69 \%)^{(68)}$. Yet, it remained much higher compared with women asylum seekers in Norway, a high-income country $(49 \%)^{(69)}$. Hanley-Cook et al. showed evidence of overreporting of food groups by $10-16 \%$ for assessing the prevalence of MDD-W of reproductive age using the listbased or open recall methods ${ }^{(74)}$. This suggests that the prevalence of LDD can be even higher in our study. Our findings are alarming and indicate that a significant proportion of women of reproductive age have a poor diet quality and are far from reaching the recommendations for nutrient requirements and dietary diversity.

FI and poor dietary diversity among mothers were significantly correlated in our study. This link has been well documented in the literature ${ }^{(63,64,75)}$. Our findings showed that a household monthly income below or approximately equal to the legal minimum wage and a higher crowding index were significantly associated with FI and LDD. It is no surprise that both indicators share common underlying determinants as a result of their leading denominator: a low socio-economic status. Similar findings were also reported by a national study among Lebanese 
Table 6. Percentage contribution of food groups to total energy intake (\% EI) by income groups based on dietary data from the FFQ (Mean values and standard deviations)

\begin{tabular}{|c|c|c|c|c|c|c|c|}
\hline \multirow[b]{3}{*}{ Food groups and subgroups ${ }^{*}$} & \multicolumn{7}{|c|}{ Percentage energy intake (\% El) } \\
\hline & \multicolumn{2}{|c|}{$\begin{array}{l}\text { Low-income group } \\
\qquad(n 267)\end{array}$} & \multicolumn{2}{|c|}{$\begin{array}{l}\text { High-income group } \\
\qquad(\text { n 142) }\end{array}$} & \multicolumn{2}{|c|}{ Total $(n$ 409) } & \multirow[b]{2}{*}{$P \dagger$} \\
\hline & Mean & SD & Mean & SD & Mean & SD & \\
\hline Grains and starchy staples & 47.6 & $16 \cdot 5$ & $40 \cdot 6$ & $14 \cdot 8$ & $45 \cdot 2$ & $16 \cdot 3$ & 0.000 \\
\hline Bread & 26.5 & 14.7 & $22 \cdot 2$ & 11.7 & $25 \cdot 0$ & 13.9 & 0.005 \\
\hline Rice & $6 \cdot 2$ & 5.5 & $5 \cdot 1$ & 3.8 & 5.8 & $5 \cdot 0$ & 0.005 \\
\hline Bulgur/pasta & 3.1 & 3.3 & $2 \cdot 6$ & $2 \cdot 2$ & 2.9 & 3.0 & 0.005 \\
\hline Potato/French fries & 11.7 & $10 \cdot 7$ & 10.5 & $8 \cdot 8$ & 11.3 & $10 \cdot 1$ & 0.005 \\
\hline Dry breadsticks & 0.1 & 0.2 & 0.2 & 0.8 & 0.1 & 0.5 & 0.005 \\
\hline Crackers/toast/cereals & 0.01 & 1.0 & 0.04 & 0.3 & 0.02 & 0.2 & 0.176 \\
\hline Vegetables & 0.9 & 0.9 & 0.9 & 0.8 & 0.9 & 0.9 & 0.574 \\
\hline Fresh vegetables & 0.8 & 0.6 & 0.9 & 0.7 & 0.8 & 0.7 & 0.197 \\
\hline Canned vegetables & 0.1 & 0.6 & 0.0 & 0.2 & 0.1 & 0.5 & 0.446 \\
\hline Fruits & 2.9 & 3.3 & 3.6 & 3.4 & $3 \cdot 2$ & 3.3 & 0.034 \\
\hline Fresh fruits & $2 \cdot 8$ & 3.2 & 3.6 & 3.3 & $3 \cdot 1$ & $3 \cdot 2$ & 0.031 \\
\hline Dried/canned/cocktails & 0.1 & 0.8 & 0.1 & 0.5 & 0.1 & 0.7 & 0.905 \\
\hline Flesh foods & $2 \cdot 1$ & $2 \cdot 0$ & $2 \cdot 6$ & $2 \cdot 8$ & $2 \cdot 3$ & $2 \cdot 3$ & 0.021 \\
\hline Beef/lamb/organ meat & 0.4 & 0.8 & 0.6 & 0.9 & 0.5 & 0.9 & 0.041 \\
\hline Processed meat & 0.3 & 0.6 & 0.2 & 0.5 & 0.3 & 0.6 & 0.446 \\
\hline Poultry & 1.1 & 1.3 & 1.4 & $2 \cdot 3$ & 1.2 & 1.7 & 0.038 \\
\hline Fish & 0.3 & 0.7 & 0.3 & 0.6 & 0.3 & 0.7 & 0.593 \\
\hline Eggs & $2 \cdot 0$ & $2 \cdot 2$ & $2 \cdot 0$ & $2 \cdot 0$ & 2.0 & $2 \cdot 2$ & 0.780 \\
\hline Pulses, nuts and seeds & $5 \cdot 1$ & $6 \cdot 1$ & 5.8 & $5 \cdot 6$ & $5 \cdot 3$ & 5.9 & 0.250 \\
\hline Legumes & $2 \cdot 7$ & $2 \cdot 6$ & $2 \cdot 8$ & $2 \cdot 7$ & $2 \cdot 7$ & $2 \cdot 6$ & 0.623 \\
\hline Nuts and seeds & $2 \cdot 4$ & $5 \cdot 4$ & 3.0 & $5 \cdot 0$ & $2 \cdot 6$ & $5 \cdot 2$ & 0.293 \\
\hline Dairy products & 5.6 & 5.6 & $6 \cdot 3$ & 4.9 & 5.8 & 5.4 & 0.182 \\
\hline Cheese & 1.6 & $2 \cdot 3$ & $2 \cdot 3$ & 2.4 & 1.8 & $2 \cdot 4$ & 0.004 \\
\hline Milk & 0.7 & 2.9 & 0.6 & 1.5 & 0.7 & 2.5 & 0.710 \\
\hline Yogurt and derivatives & 3.3 & 3.7 & 3.4 & 3.6 & 3.3 & 3.7 & 0.721 \\
\hline Mixed dishes & $2 \cdot 4$ & $2 \cdot 2$ & $2 \cdot 2$ & $2 \cdot 3$ & $2 \cdot 3$ & $2 \cdot 2$ & 0.443 \\
\hline Pizzas and pies & $2 \cdot 1$ & $3 \cdot 4$ & $2 \cdot 4$ & 3.0 & $2 \cdot 2$ & 3.3 & 0.406 \\
\hline Fats and oils & $15 \cdot 0$ & 11.8 & $16 \cdot 9$ & 11.4 & $15 \cdot 7$ & 0.6 & 0.116 \\
\hline Olive oil & $3 \cdot 3$ & 4.2 & 4.8 & 5.3 & $3 \cdot 8$ & 4.7 & 0.002 \\
\hline Other vegetable oils & 9.5 & 9.5 & $9 \cdot 3$ & 8.9 & 9.4 & $9 \cdot 3$ & 0.852 \\
\hline Other fats & $2 \cdot 2$ & $6 \cdot 2$ & $2 \cdot 8$ & 4.6 & 2.4 & 5.7 & 0.338 \\
\hline Sweets and sugar derivatives & $7 \cdot 3$ & $6 \cdot 3$ & 8.8 & $6 \cdot 1$ & 7.8 & $6 \cdot 3$ & 0.020 \\
\hline Sugar/jam/honey/molasses & $2 \cdot 8$ & 2.9 & 2.8 & $2 \cdot 7$ & 2.8 & $2 \cdot 8$ & 0.869 \\
\hline Desserts/pastries/ice cream/puddings & $2 \cdot 8$ & 4.3 & 3.4 & 3.8 & $3 \cdot 1$ & $4 \cdot 1$ & 0.135 \\
\hline Chocolate/candies & 1.7 & 3.5 & 2.6 & 3.8 & $2 \cdot 0$ & 3.6 & 0.014 \\
\hline Salty snacks/potato chips & $2 \cdot 4$ & 4.9 & $2 \cdot 7$ & 3.8 & 2.5 & 4.6 & 0.552 \\
\hline All beverages & $2 \cdot 6$ & $2 \cdot 9$ & $2 \cdot 9$ & $2 \cdot 6$ & $2 \cdot 7$ & $2 \cdot 8$ & 0.370 \\
\hline Tea & 0.09 & 0.1 & 0.07 & 0.1 & 0.08 & 0.1 & 0.015 \\
\hline Coffee & 0.1 & 0.2 & 0.1 & $0 \cdot 1$ & 0.1 & 0.1 & 0.228 \\
\hline Sugar-sweetened beverages & $2 \cdot 4$ & $2 \cdot 9$ & $2 \cdot 7$ & $2 \cdot 6$ & 2.5 & $2 \cdot 8$ & 0.392 \\
\hline Other: alcoholic/diet soda & 0.0 & 0.1 & 0.0 & 0.2 & 0.0 & 0.2 & 0.259 \\
\hline Condiments and pickles & 1.8 & $2 \cdot 4$ & 1.5 & 1.8 & $1 \cdot 7$ & $2 \cdot 2$ & 0.185 \\
\hline
\end{tabular}

$\% \mathrm{El}$, percentage energy intake.

* Continuous variables are expressed as mean values and standard deviations.

† Significantly different at $P$-value $<0.05 ; P$-value was derived using one-way ANOVA test.

mothers ${ }^{(73)}$. Furthermore, receiving WFP food assistance was significantly associated with LDD among Syrian refugee mothers in Greater Beirut in our study. This suggests that WFP food assistance may not necessarily ensure a diverse diet. Staple foods accounted for $42 \%$ of total food expenditure among Syrian refugees in Lebanon, while only $13 \%$ was spent on fruits and vegetables and $8 \%$ on fresh meat ${ }^{(70)}$. This indicates that a more monotonous diet was consumed by the refugees. In fact, Beirut witnessed a drawback in food consumption scores and Mount Lebanon continued to have the poorest food consumption scores, despite a nationwide improvement in poor and borderline food consumption levels among Syrian refugees in
Lebanon in 2018-2019(26). Moreover, poor dietary diversity and low meal frequency emerged as potential coping mechanisms against FI among mothers in our study. Syrian refugees, notably in Mount Lebanon, were increasingly reducing the number of meals eaten per day and portion sizes but also restricting food consumption by adults for the sake of children ${ }^{(26)}$.

Our previous study showed evidence of higher proportions of nutritional inadequacies among PM and LM as well as an overall maternal diet rich in sugar and fat and poor in protein, fibres and micronutrients ${ }^{(28)}$. Further analysis showed that the consumption of food groups was also consistent with the 'Western diet' in the present study, characterising the nutrition 
transition in low- and middle-income countries ${ }^{(76)}$. Overall, high contributions to energy intake from grains, starchy staples, and oils and fats were recorded over the past year with poor incorporation of nutrient-rich foods. Contribution of legumes, nuts, seeds, fruits and vegetables was low, particularly of vitamin Arich fruits and green leafy vegetables. Similar nutritional habits and monotonous diets were found among asylum seekers living in Germany ${ }^{(77,78)}$ and Norway ${ }^{(69)}$. Nasreddine et al. ${ }^{(79)}$ also highlighted a shift in consumption towards refined grains, highly processed foods and added fats and oils and deviating from nutritious foods in Lebanon. Food systems and environments are well-known to influence food choices, customs and diets as well as to a great extent food security and nutrition. Key aspects include the physical, economic, political and socio-cultural context. Food customs are also shaped by religions and beliefs, traditions, cultures and social norms ${ }^{(80)}$. According to a recent study, food played a central role in the social integration among Syrian refugees in Germany and was considered essential to their cultural value and psychological well-being despite challenges associated with food-related autonomy and environment. However, the authors also highlighted that, while Syrian refugees in the Middle East might benefit from a similar food environment to the one in Syria, refugees in Lebanon might be confronted with higher economic hardships hindering their purchasing power and access to food ${ }^{(81)}$.

Socio-economic disparities, such as the income, shape the diet structure to a great extent among vulnerable populations. Higher contributions to energy intake from grains and refined starchy staples such as bread and rice were found among lowincome households in our findings. Lower income groups usually spend a higher share of money on food, influencing their food choices. The higher cost of a healthy diet may be a significant barrier, especially that lean meats, fish and fresh produce are more expensive energy sources compared with grains, fats and sweets ${ }^{(82)}$. Syrian refugee households in Mount Lebanon recorded the highest percentage of food expenditures on bread and pasta (47\%) compared with other governorates in Lebanon ${ }^{(70)}$. Inversely, high-income households in our study had a more diverse diet with significantly higher contributions of fruits, cheese, olive oil and flesh foods but also of sweets and sugar derivatives to total energy consumption. Lopez et al. ${ }^{(83)}$ also found that other vegetable oils were consumed more than olive oil to lower the total costs of the diet. A Mediterranean dietary pattern was found to be more expensive than a Western dietary pattern, as it is mostly based on the consumption of local fruits and vegetables, beans, nuts and seeds, dairy products and olive oil ${ }^{(84)}$. With increasing economic vulnerabilities and FI, poor diet quality and diversity are often the result of an increased consumption of cheap and high energy-dense foods rather than nutrient-dense foods as a strategy to stretch the energy cost and reduce food expenditures ${ }^{(82)}$. An inadequate intake of vitamins and minerals and distorted contribution of fats and sugars to energy can lead to under- and overnutrition alongside persisting micronutrient deficiencies ${ }^{(15,85,86)}$. The cooccurrence of overnutrition and anaemia among mothers and undernutrition among children was reported in our previous study among Syrian refugees from the same household in Greater Beirut ${ }^{(28)}$.
Regarding the mental health status of the mothers, nearly one-third were suffering from PTSD and/or moderate to severe depression in our study. Comparable and higher prevalence rates were reported among Palestinian and Syrian refugees in Lebanon ${ }^{(72,87-90)}$. A strong relationship was observed between poor maternal mental health, FI and a high crowding index in our findings. Poverty and FI are well documented as factors associated with psychological distress and depression symptoms. The struggle to provide food on a day-to-day basis and worrying about living expenses can exacerbate effects on mental health ${ }^{(18,91)}$. Certainly, overcrowding, poor living conditions and lack of resources can accumulate the risk of deterioration of the health status ${ }^{(35)}$. Furthermore, evidence on the association between depression, FI and poor dietary diversity is found in the literature, suggesting a relationship between micronutrient deficiencies and mental health disorders ${ }^{(17,92,93)}$. However, the lack of association between the mental health status and LDD in our study could be due to the fact that the MDD-W indicator does not assess the intake of certain nutrients or food groups related to mental health outcomes.

As for the nutritional status of the mothers, the lack of association with LDD and FI observed in our survey was in line with the literature ${ }^{(73,94-96)}$, even though a relationship between FI and obesity was documented among Lebanese mothers ${ }^{(56)}$. One explanation could be that the food group diversity score does not account for the amounts of food consumed, physical activity levels and total energy expenditure ${ }^{(94)}$. We also suggest it could be explained by the exclusion of the nutrient-dense food groups from the score, such as oils and fats, sweets, snacks and sugarsweetened beverages. Furthermore, anaemia was not associated with maternal LDD and FI in our survey, similar to findings from pregnant women in Ghana ${ }^{(97)}$ and Pakistan ${ }^{(98)}$. Other studies were able to demonstrate a relationship between anaemia and LDD as well as $\mathrm{FI}^{(99,100)}$. The multifactorial nature of anaemia can provide an explanation to these controversial findings as several factors may act as causes or contributors. These may be related not only to poor dietary practices, low bioavailability of nutrients, high intake of inhibitors, genetic disorders, infections and inflammation but also to environmental and socio-economic factors including women's empowerment, poverty, education and access to healthcare ${ }^{(101)}$. In fact, a very plausible explanation regarding the lack of association in our study could be related to the role of health and nutrition awareness programmes. While our previous study showed that receiving health and nutrition messages and access to the free or subsidised healthcare in place for refugees in Lebanon could have played a role in reducing anaemia ${ }^{(28)}$, our present findings also shed light on the role of healthcare professionals. Once more, an indication on the beneficial impact of health services offered to refugees is found and emphasises the crucial role of counselling on improving dietary diversity and anaemia.

New threats to food security and nutrition were rising in Lebanon since October 2019 due to the strong socio-political unrest and severe financial crisis involving spiralling food inflation rates and strong currency depreciation. With the combination of containment measures for the COVID-19 pandemic, unemployment rates and reduced income soared across Lebanon for the population and refugees ${ }^{(102)}$. In addition, 
Lebanon relies heavily on imported commodities and food to cover domestic needs; for instance, $80 \%$ of cereals consumption is met through imports. Long-term effects of the Beirut port explosion on 4 August 2020 will reflect on the economy and supply chain, particularly in Beirut and Mount Lebanon ${ }^{(103)}$. As the ability to access and purchase food is compromised even further, food security and nutrition are expected to worsen and the risk of deterioration of the health and nutritional status of vulnerable groups is higher among both the host population and refugees in Lebanon ${ }^{(25)}$.

Double-duty actions and multicomponent strategies targeting multiple forms of malnutrition simultaneously are a priority and need to tackle social determinants of health and nutrition disparities $^{(86)}$. Economic interventions, subsidies and fiscal incentives should be considered to encourage changes in dietary behaviours towards a healthier and sustainable diet. For instance, financial and food assistance programmes should be strengthened and provide additional support to the most vulnerable groups among refugees and the host population. Our findings also shed light on the crucial role of a multidisciplinary approach with a diverse team of healthcare professionals to improve nutrition knowledge, family planning and mental health of women of reproductive age through counselling and awareness-raising sensitive to cultural norms. In light of increasing vulnerabilities, Syrian refugees lack resources to cover essential needs ${ }^{(25)}$. Admittedly, recent studies showed that Syrian women are willing to overcome sex barriers and cultural norms to accessing economic participation when working conditions are decent and safe ${ }^{(104)}$. Policy efforts should target to improve women's access to economic resources. Strategies need to consider the role of women as both a caregiver and a provider to combat FI and LDD among mothers in poor and vulnerable settings ${ }^{(105)}$.

\section{Limitations}

Few limitations should be considered when interpreting the study findings. First, the design of the study is cross-sectional; hence, it cannot infer causality but rather associational relationships. The study was narrowed to the most vulnerable areas of Greater Beirut and to mothers attending PHCC. This may limit the findings to be representative of Syrian refugees in rural settings and at a national level. Recruitment took place in Greater Beirut, covering Beirut and adjacent districts of Mount Lebanon. It is considered to be the melting pot of the country with more than half of the Lebanese population and over 305000 refugees $^{(30,106)}$. Second, our measure of FI at the individual level could limit the comparison with other findings measuring household FI using different scales. However, the Food Insecurity Experience Scale has demonstrated its robustness and provides the opportunity to generate internationally comparable measures of $\mathrm{FI}^{(107)}$. Third, the Patient Health Questionnaire-9 was shown to have a good sensitivity but poor specificity in capturing depressive symptoms among adults in Lebanon. The tool was found to be useful in screening for depression in settings lacking sufficient psychiatric care to determine the need for psychiatry referral ${ }^{(33)}$. Lastly, dietary data may be subjected to possible recall bias. Enumerators underwent extensive trainings and used standardised protocols to limit the social desirability bias and overcome the memory bias. The 24-h dietary call was collected using the five-step multiple pass recall method, which may improve the accuracy of dietary estimates among adults; however, underreporting under field conditions and among overweight and obese adults remains a constraint ${ }^{(48,108,109)}$. The 24-h dietary recall may not take into account seasonal variations; however, the latter is compensated for by the use of FFQ over the past 12 months $^{(110)}$. The FFQ used in our study encompassed a wide range of food groups, including ultra-processed foods. This is an advantage in measuring changing dietary patterns in the context of the nutrition transition ${ }^{(111)}$. Another limitation worth mentioning is the absence of an extensive food composition database in Lebanon. So, the USDA database was used, and specific food items were added from the local food composition tables available for a limited number of local foods. Although the absolute estimates of dietary intake could have been affected, its effects on the observed inadequate intakes among mothers would have been limited.

\section{Conclusion}

The present study shows evidence of poor dietary diversity and mental health as well as FI among Syrian refugee mothers in an urban setting of a humanitarian crisis. Findings demonstrate poor nutritional practices and low diet quality among women of reproductive age. With increasing economic vulnerabilities and FI, inadequate dietary habits often take place and compromise the quality of the diet. Multifaceted interventions are needed to address the situation holistically, including nutrition-specific interventions and assistance programmes, which can be developed or strengthened at the local and national level, in addition to psychosocial support and family planning. Both, the women's roles as a caregiver and as provider need to be considered in order to reduce FI and improve dietary diversity among vulnerable groups. Further research is needed to examine the impact of suboptimal maternal nutrition during the first $1000 \mathrm{~d}$ on the diet quality and health of children in a humanitarian context. The relationship between poor maternal mental health, breast-feeding practices and maternal nutrition needs to be further explored among Syrian refugee mothers in Lebanon.

\section{Acknowledgements}

The authors are deeply grateful to all the participants for participating in the survey. We would like to thank the directors of primary health care centres for granting us access to their premises to conduct the interviews. The centres included Howard Karagheusian Commemorative Corporation and Armenian Relief Cross of Lebanon in Bourj Hammoud, Makhzoumi Foundation PHC in Mazraa, Child \& Mother Welfare Hospital in Msaytbeh, Mar Antonious PHC in Baouchriyeh and Maternal Childhood Center in Chiyah and Bourj Barajneh. We would like to extend our gratitude and deep appreciation to our volunteers, field workers and research assistants for their hard work during data collection. We are sincerely grateful to the Medical Research Volunteer Program for their supply of assistance during data 
collection and entry. We would like to acknowledge the help of Dr Yitbarek Kidane Woldetensay for advancing the analysis of mental health indicators. Last but not least, we would like to extend our thanks to Hisham Kanaan for his support in the use of statistical software.

The publication is an output of a PhD scholarship from the Food Security Center from the University of Hohenheim which is part of the DAAD (German Academic Exchange Service) programme 'exceed' and is supported by DAAD and the German Federal Ministry for Economic Cooperation and Development (BMZ) and in cooperation with the hosting Institute of Nutritional Sciences (140). Support was also received by the Fiat Panis Foundation, the BCFN YES! 2017 Research Grant Award from the Barilla Center for Food \& Nutrition (BCFN) Foundation and by the University Research Board at the American University of Beirut (award number: 103366). The funding organisations had no role in the design, data collection, analysis or writing of this manuscript.

J. A., T. J. and V. S. conceptualised the research design and led the parent study. L. N., L. J. and N. H. provided support in designing the parent study. J. A., T. J., V. S. and L. N. sought after ethics approval. J. A. and T. J. organised, implemented and supervised the study during data collection and entry. J. A. conducted data analysis, conceptualised and wrote the original draft of the manuscript. G. C. supported data analysis. T. J., V. S., J. F., L. N., L. J. and N. H. critically reviewed the manuscript. All authors have read and agreed to the published version of this manuscript.

The authors declare no conflict of interest.

\section{Supplementary material}

For supplementary material referred to in this article, please visit https://doi.org/10.1017/S0007114521004724

\section{References}

1. United Nations (2020) The Sustainable Development Goals Report 2020. New York, NY: UN.

2. Food and Agriculture Organization (1996) Rome Declaration on World Food Security and World Food Summit Plan of Action: World Food Summit, 13-17 November 1996, Rome, Italy. Rome: FAO.

3. Food and Agriculture Organization (2017) Regional Overview of Food Security and Nutrition - Building Resilience for in Times of Conflict and Crisis: Food Security and Nutrition a Perspective from the Near East and North Africa (NENA) Region. Cairo: FAO.

4. von Grebmer K, Bernstein J, Hammond L, et al. (2018) 2018 Global Hunger Index: Forced Migration and Hunger. Bonn: Welthungerhilfe and Concern Worldwide.

5. Food and Agriculture Organization, International Fund for Agricultural Development, United Nations Children's Fund, et al. (2020) The State of Food Security and Nutrition in the World 2020. Transforming Food Systems for Affordable Healthy Diets. Rome: FAO.

6. Food and Agriculture Organization, International Fund for Agricultural Development, United Nations Children's Fund, et al. (2019) The State of Food Security and Nutrition in the
World 2019. Safeguarding against Economic Slowdowns and Downturns. Rome: FAO.

7. Ghattas H (2014) Food Security and Nutrition in the Context of the Global Nutrition Transition. Rome: FAO.

8. Government of Lebanon \& the United Nations (2020) Lebanon Crisis Response Plan 2017-2020 (2020 Update). Lebanon: Government of Lebanon \& the United Nations.

9. Maxwell D \& Caldwell R (2008) Coping Strategies Index: Field Methods Manual, 2nd ed. Geneva: Cooperative for Assistance and Relief Everywhere, Inc.

10. Ballard T, Kepple A \& Cafiero C (2013) The Food Insecurity Experience Scale - Development of a Global Standard for Monitoring Hunger Worldwide. Rome: Food and Agriculture Organization.

11. Food and Agriculture Organization (2021) Minimum Dietary Diversity for Women: An Updated Guide to Measurement from Collection to Action. Rome: FAO.

12. Langley-Evans SC (2015) Nutrition in early life and the programming of adult disease: a review. J Hum Nutr Diet 28, $1-14$.

13. Black RE, Victora CG, Walker SP, et al. (2013) Maternal and child undernutrition and overweight in low-income and middle-income countries. Lancet 382, 427-451.

14. Christian P \& Stewart CP (2010) Maternal micronutrient deficiency, fetal development, and the risk of chronic disease. $J$ Nutr 140, 437-445.

15. Tanumihardjo SA, Anderson C, Kaufer-Horwitz M, et al. (2007) Poverty, obesity, and malnutrition: an international perspective recognizing the paradox. J Am Diet Assoc 107, 1966-1972.

16. Drewnowski A (2007) The real contribution of added sugars and fats to obesity. Epidemiol Rev 29, 160-171.

17. Sparling TM, Waid JL, Wendt AS, et al. (2020) Depression among women of reproductive age in rural Bangladesh is linked to food security, diets and nutrition. Public Health Nutr 23, 660-673.

18. Emerson JA, Tol W, Caulfield LE, et al. (2017) Maternal psychological distress and perceived impact on child feeding practices in South Kivu, DR Congo. Food Nutr Bull 38, 319-337.

19. Stein A, Malmberg L-E, Sylva K, et al. (2008) The influence of maternal depression, caregiving, and socioeconomic status in the post-natal year on children's language development. Child Care Health Dev 34, 603-612.

20. Trapolini T, Ungerer JA \& McMahon CA (2008) Maternal depression: relations with maternal caregiving representations and emotional availability during the preschool years. Attach Hum Dev 10, 73-90.

21. Woldetensay YK, Belachew T, Ghosh S, et al. (2021) The effect of maternal depressive symptoms on infant feeding practices in rural Ethiopia: community based birth cohort study. Int Breastfeed J 16, 27.

22. Breisinger C, Ecker O, Al-Riffai P, et al. (2012) Beyond the Arab Awakening: Policies and Investments for Poverty Reduction and Food Security. Washington, DC: International Food Policy Research Institute.

23. Quber M \& Moussa SS (2018) Arab spring as a background of civil war in Syria. Int Conf Knowl Based Organ 24, 245-251.

24. United Nations High Commissioner for Refugees (2021) Situation Syria Regional Refugee Response. https://data2. unhcr.org/en/situations/syria (accessed September 2021).

25. United Nations High Commissioner for Refugees, United Nations Children's Fund \& World Food Programme (2021) VASYR 2020 - Vulnerability Assessment of Syrian Refugees in Lebanon. Lebanon: UNHCR. 
26. United Nations High Commissioner for Refugees, United Nations Children's Fund \& World Food Programme (2019) VASYR 2019 - Vulnerability Assessment of Syrian Refugees in Lebanon. Lebanon: UNHCR.

27. United Nations Office for the Coordination of Humanitarian Affairs (2014) Lebanon: Beirut and Mount Lebanon Governorate Profile (as of 4 March 2014). Lebanon: OCHA.

28. Abou-Rizk J, Jeremias T, Nasreddine L, et al. (2021) Anemia and nutritional status of Syrian refugee mothers and their children under 5 years in greater Beirut, Lebanon. Int J Environ Res Public Health 18, 6894.

29. United Nations High Commissioner for Refugees (2015) Most Vulnerable Localities in Lebanon - March 2015. Lebanon: UNHCR.

30. United Nations Office for the Coordination of Humanitarian Affairs (2016) Lebanon: Beirut and Mount Lebanon Governorates Profile (May 2016). Lebanon: OCHA.

31. United Nations Children's Fund (2014) 2013 Joint Nutrition Assessment Syrian Refugees in Lebanon. Lebanon: UNICEF.

32. Kroenke K, Spitzer RL \& Williams JB (2001) The PHQ-9: validity of a brief depression severity measure.J Gen Intern Med 16, 606-613.

33. Sawaya H, Atoui M, Hamadeh A, et al. (2016) Adaptation and initial validation of the Patient Health Questionnaire -9 (PHQ9) and the generalized anxiety disorder -7 questionnaire (GAD-7) in an Arabic speaking Lebanese psychiatric outpatient sample. Psychiatry Res 239, 245-252.

34. Kadri N, Agoub M, El Gnaoui S, et al. (2005) Moroccan colloquial Arabic version of the mini international neuropsychiatric interview (MINI): qualitative and quantitative validation. Eur Psychiatry 20, 193-195.

35. World Health Organization (2018) WHO Housing and Health Guidelines. Geneva: WHO

36. International Labour Organization (2014) Syrian Refugees in Lebanon Face Harsh Working Conditions. http://www.ilo. org/global/about-the-ilo/newsroom/news/WCMS_240126/ lang-en/index.htm (accessed August 2020).

37. Food and Agriculture Organization (2018) SDG Indicator 2.1.2 - Using the Food Insecurity Experience Scale (FIES) Lesson 1: Introduction to the Food Insecurity Experience Scale. Rome: FAO.

38. Food and Agriculture Organization (2018) SDG Indicator 2.1.2 - Using the Food Insecurity Experience Scale (FIES) Lesson 3: Statistical Validation of FIES Data. Rome: FAO.

39. Cafiero C, Viviani S \& Nord M (2018) Food security measurement in a global context: the food insecurity experience scale. Measurement 116, 146-152.

40. Centers for Disease Control and Prevention (2017) National Health and Nutrition Examination Survey (NHANES) Anthropometry Procedures Manual. Hyattsville, MD: CDC.

41. Fakier A, Petro G \& Fawcus S (2017) Mid-upper arm circumference: a surrogate for body mass index in pregnant women. $S$ Afr Med J 107, 606-610.

42. Tumilowicz A (2010) Guide to Screening for Food and Nutrition Services Among Adolescents and Adults Living with $H I V$. Washington, DC: Food and Nutrition Technical Assistance III Project (FANTA), FHI 360.

43. Food and Nutrition Technical Assistance, United States Agency for International Development \& FHI 360 (2018) Global MUAC Cutoffs for Adults: A Technical Consultation. Washington, DC: FANTA Project.

44. United Nations High Commissioner for Refugees \& World Food Programme (2011) Guidelines for Selective Feeding: The Management of Malnutrition in Emergencies. Geneva: UNHCR.
45. National Institutes of Health (1998) Clinical guidelines on the identification, evaluation, and treatment of overweight and obesity in adults - the evidence report. Obes Res $\mathbf{6}$, Suppl. 2, 51S-209S.

46. World Health Organization (2011) Haemoglobin Concentrations for the Diagnosis of Anaemia and Assessment of Severity. Vitamin and Mineral Nutrition Information System. Geneva: WHO.

47. Millen BE \& Morgan JL (1996) The 2D Food Portion Visual. Framingham, MA: Nutrition Consulting Enterprises.

48. Conway JM, Ingwersen LA \& Moshfegh AJ (2004) Accuracy of dietary recall using the USDA five-step multiple-pass method in men: an observational validation study. J Am Diet Assoc 104, 595-603.

49. Johnson RK (2002) Dietary intake - how do we measure what people are really eating? Obes Res 10, Suppl. 1, 63S-68S.

50. El Sayed Ahmad R, Baroudi M, Shatila H, et al. (2020) Validity and reproducibility of a culture-specific food frequency questionnaire in Lebanon. Nutrients 12, 3316.

51. Pellett PL \& Shadarevian S (2013) Food Composition: Tables for Use in the Middle East. Beirut: American University of Beirut Press.

52. Issa L, Alghanim F \& Obeid N (2018) Analysis of food pairing in some eastern Mediterranean countries. In 2018 8th International Conference on Computer Science and Information Technology (CSIT), pp. 167-172.

53. Erhardt J (2010) Nutrition Surveys and Assessment NutriSurvey Software 2007. http://www.nutrisurvey.de/ index.html (accessed August 2020).

54. Institute of Medicine (2011) Dietary Reference Intakes for Calcium and Vitamin D. Washington, DC: National Academies Press (US).

55. Ponza M, Ohls J, Millen B, et al. (1996) Serving Elders at Risk. The Older Americans Act Nutrition Programs: National Evaluation of the Elderly Nutrition Program 1993-1995. Volume I: Title III Evaluation Findings. Princeton, NJ: Mathematica Policy Research, US Department of Health and Human Services, Office of the Assistant Secretary for Aging.

56. Jomaa L, Naja F, Cheaib R, et al. (2017) Household food insecurity is associated with a higher burden of obesity and risk of dietary inadequacies among mothers in Beirut, Lebanon. BMC Public Health 17, 567.

57. Nasreddine L, Chamieh MC, Ayoub J, et al. (2020) Sex disparities in dietary intake across the lifespan: the case of Lebanon. Nutr J 19, 24.

58. Hwalla N, Adra N \& Jackson RT (2004) Iron deficiency is an important contributor to anemia among reproductive age women in Lebanon. Ecol Food Nutr 43, 77-92.

59. Harvard Humanitarian Initiative (2012) KoBoToolbox - Data Collection Tools for Challenging Environments. https:// kobotoolbox.org/ (accessed August 2020).

60. Chakona G \& Shackleton C (2017) Minimum dietary diversity scores for women indicate micronutrient adequacy and food insecurity status in South African towns. Nutrients $\mathbf{9}, 812$.

61. Custodio E, Kayikatire F, Fortin S, et al. (2020) Minimum dietary diversity among women of reproductive age in urban Burkina Faso. Matern Child Nutr 16, e12897.

62. Desta M, Akibu M, Tadese M, et al. (2019) Dietary diversity and associated factors among pregnant women attending antenatal clinic in Shashemane, Oromia, Central Ethiopia: a cross-sectional study. J Nutr Metab 2019, 3916864.

63. Kang Y, Hurley KM, Ruel-Bergeron J, et al. (2019) Household food insecurity is associated with low dietary diversity among pregnant and lactating women in rural Malawi. Public Health Nutr 22, 697-705. 
64. Singh DR, Ghimire S, Upadhayay SR, et al. (2020) Food insecurity and dietary diversity among lactating mothers in the urban municipality in the mountains of Nepal. PLOS ONE 15, e0227873.

65. Yeneabat T, Adugna H, Asmamaw T, et al. (2019) Maternal dietary diversity and micronutrient adequacy during pregnancy and related factors in East Gojjam Zone, Northwest Ethiopia, 2016. BMC Pregnancy Childbirth 19, 173.

66. Jemal K \& Awol M (2019) Minimum dietary diversity score and associated factors among pregnant women at Alamata general hospital, Raya Azebo Zone, Tigray Region, Ethiopia. J Nutr Metab 2019, 8314359.

67. Aliwo S, Fentie M, Awoke T, et al. (2019) Dietary diversity practice and associated factors among pregnant women in North East Ethiopia. BMC Res Notes 12, 123.

68. Morseth MS, Grewal NK, Kaasa IS, et al. (2017) Dietary diversity is related to socioeconomic status among adult Saharawi refugees living in Algeria. BMC Public Health 17, 621.

69. Henjum S, Caswell BL \& Terragni L (2019) 'I feel like I'm eating rice $24 \mathrm{~h}$ a day, 7 days a week': dietary diversity among asylum seekers living in Norway. Nutrients 11, 2293.

70. United Nations High Commissioner for Refugees, United Nations Children's Fund \& World Food Programme (2018) VASYR 2018 - Vulnerability Assessment of Syrian Refugees in Lebanon. Lebanon: UNHCR

71. Ghattas H, Sassine AJ, Seyfert K, et al. (2014) Food insecurity among Iraqi refugees living in Lebanon, 10 years after the invasion of Iraq: data from a household survey. BrJ Nutr 112, 70-79.

72. Ghattas H, Sassine AJ, Seyfert K, et al. (2015) Prevalence and correlates of food insecurity among Palestinian refugees in Lebanon: data from a household survey. PLOS ONE 10, e0130724.

73. Jomaa LH, Naja FA, Kharroubi SA, et al. (2020) Food insecurity is associated with compromised dietary intake and quality among Lebanese mothers: findings from a national cross-sectional study. Public Health Nutr 23, 2687-2699.

74. Hanley-Cook GT, Tung JYA, Sattamini IF, et al. (2020) Minimum dietary diversity for women of reproductive age (MDD-W) data collection: validity of the list-based and open recall methods as compared to weighed food record. Nutrients 12, 2039.

75. Na M, Mehra S, Christian P, et al. (2016) Maternal dietary diversity decreases with household food insecurity in rural Bangladesh: a longitudinal analysis. J Nutr 146, 2109-2116.

76. Popkin BM, Adair LS \& Ng SW (2012) Global nutrition transition and the pandemic of obesity in developing countries. Nutr Rev 70, 3-21.

77. Khan S, Fischer L, Ghaziani S, et al. (2019) Nutritional habits of asylum seekers living in communal accommodation in Stuttgart, Germany. Ernaehr Umsch 66, 18-25.

78. Schmitt R, Fülle J, Abou-Rizk J, et al. (2019) Nutritional habits of female asylum seekers - from tradition to adaptation. Ernaehr Umsch 66, 45-51.

79. Nasreddine L, Ayoub JJ, Hachem F, et al. (2019) Differences in dietary intakes among Lebanese adults over a decade: results from two national surveys 1997-2008/2009. Nutrients 11, 1738.

80. HLPE (2017) Nutrition and Food Systems. A Report by the High Level Panel of Experts on Food Security and Nutrition of the Committee on World Food Security. Rome: FAO.

81. Al-Sayed L \& Bieling C (2021) Understanding food-related well-being in a diaspora situation: the psychological and social dimensions. J Refug Stud, feab069. https://academic-oup- com.eres.qnl.qa/jrs/advance-article-abstract/doi/10.1093/jrs/ feab069/6242744?redirectedFrom=fulltext.

82. Darmon N \& Drewnowski A (2015) Contribution of food prices and diet cost to socioeconomic disparities in diet quality and health: a systematic review and analysis. Nutr Rev $\mathbf{7 3}$, 643-660

83. Lopez CN, Gonzalez MA, Villegas A, et al. (2009) Costs of Mediterranean and western dietary patterns in a Spanish cohort and their relationship with prospective weight change. J Epidemiol Community Health 63, 920-927.

84. Hachem F, Capone R, Yannakoulia M, et al. (2016) The Mediterranean diet: a sustainable consumption pattern. In Mediterra 2016. Zero Waste in the Mediterranean. Natural Resources, Food and Knowledge/International Centre for Advanced Mediterranean Agronomic Studies (CIHEAM) and Food and Agriculture Organization of the United Nations (FAO), pp. 243-262. Paris: Presses de Sciences Po.

85. Hwalla N, Al Dhaheri AS, Radwan H, et al. (2017) The prevalence of micronutrient deficiencies and inadequacies in the middle east and approaches to interventions. Nutrients 9, 229

86. Perez-Escamilla R, Bermudez O, Buccini GS, et al. (2018) Nutrition disparities and the global burden of malnutrition. BMJ 361, k2252.

87. Hyland P, Ceannt R, Daccache F, et al. (2018) Are posttraumatic stress disorder (PTSD) and complex-PTSD distinguishable within a treatment-seeking sample of Syrian refugees living in Lebanon? Glob Ment Health 5, e14.

88. Kazour F, Zahreddine NR, Maragel MG, et al. (2017) Post-traumatic stress disorder in a sample of Syrian refugees in Lebanon. Compr Psychiatry 72, 41-47.

89. Stevenson K, Alameddine R, Rukbi G, et al. (2019) High rates of maternal depression amongst Syrian refugees in Lebanon a pilot study. Sci Rep 9, 11849.

90. Naal H, Nabulsi D, El Arnaout N, et al. (2021) Prevalence of depression symptoms and associated sociodemographic and clinical correlates among Syrian refugees in Lebanon. BMC Public Health 21, 217.

91. Elgar FJ, Pickett W, Pförtner T-K, et al. (2021) Relative food insecurity, mental health and wellbeing in 160 countries. Soc Sci Med 268, 113556.

92. Poorrezaeian M, Siassi F, Milajerdi A, et al. (2017) Depression is related to dietary diversity score in women: a cross-sectional study from a developing country. Ann Gen Psychiatry 16, 39

93. Woldetensay YK, Belachew T, Biesalski HK, et al. (2018) The role of nutrition, intimate partner violence and social support in prenatal depressive symptoms in rural Ethiopia: community based birth cohort study. BMC Pregnancy Childbirth 18, 374 .

94. Gómez G, Fisberg RM, Nogueira Previdelli Á, et al. (2019) Diet quality and diet diversity in eight Latin American countries: results from the Latin American study of nutrition and health (ELANS). Nutrients 11, 1605.

95. Salehi-Abargouei A, Akbari F, Bellissimo N, et al. (2016) Dietary diversity score and obesity: a systematic review and meta-analysis of observational studies. Eur I Clin Nutr 70, 1-9.

96. Kehoe SH, Wrottesley SV, Ware L, et al. (2021) Food insecurity, diet quality and body composition: data from the healthy life trajectories initiative (HeLTI) pilot survey in urban Soweto, South Africa. Public Health Nutr 24, 1-9.

97. Saaka M, Oladele J, Larbi A, et al. (2017) Dietary diversity is not associated with haematological status of pregnant women resident in rural areas of Northern Ghana. J Nutr Metab 2017 , 8497892 . 
98. Ali F, Thaver I \& Khan SA (2014) Assessment of dietary diversity and nutritional status of pregnant women in Islamabad, Pakistan. J Ayub Med Coll Abbottabad 26, 506-509.

99. Ghose B, Tang S, Yaya S, et al. (2016) Association between food insecurity and anemia among women of reproductive age. PeerJ 4, e1945.

100. Zerfu TA, Umeta M \& Baye K (2016) Dietary diversity during pregnancy is associated with reduced risk of maternal anemia, preterm delivery, and low birth weight in a prospective cohort study in rural Ethiopia. Am J Clin Nutr 103, 1482-1488.

101. World Health Organization (2020) United Nations Decade of Action on Nutrition. http://www.who.int/nutrition/decadeof-action/en/ (accessed December 2020).

102. World Food Programme (2020) Assessing the Impact of the Economic and COVID-19 Crises in Lebanon - June 2020. Lebanon: WFP.

103. World Food Programme (2020) Beirut Port Explosion: Impact on Key Economic and Food Security Indicators - August 2020. Lebanon: WFP.

104. United Nations Entity for Gender Equality and the Empowerment of Women (2019) Document - Lebanon: UN Women - Addressing Gender amongst Syrian Refugees in Lebanon - September 2019. Lebanon: UN Women.
105. Schmeer KK, Piperata BA, Herrera Rodríguez A, et al. (2015) Maternal resources and household food security: evidence from Nicaragua. Public Health Nutr 18, 2915-2924.

106. United Nations Office for the Coordination of Humanitarian Affairs (2016) Lebanon Overview (May 2016). Lebanon: OCHA.

107. Saint Ville A, Po JYT, Sen A, et al. (2019) Food security and the Food Insecurity Experience Scale (FIES): ensuring progress by 2030. Food Sec 11, 483-491.

108. Conway JM, Ingwersen LA, Vinyard BT, et al. (2003) Effectiveness of the US Department of Agriculture 5-step multiple-pass method in assessing food intake in obese and non-obese women. Am J Clin Nutr 77, 1171-1178.

109. Moshfegh AJ, Rhodes DG, Baer DJ, et al. (2008) The US department of agriculture automated multiple-pass method reduces bias in the collection of energy intakes. Am J Clin Nutr 88, 324-332.

110. Food and Agriculture Organization (2018) Dietary Assessment: A Resource Guide to Method Selection and Application in Low Resource Settings. Rome: FAO.

111. Walls HL, Johnston D, Mazalale J, et al. (2018) Why we are still failing to measure the nutrition transition. BMJ Glob Health $\mathbf{3}$, e000657. 